\title{
Extraction-Separation Performance and Dynamic Modeling of Orion Test Vehicles with Adams Simulation: $2^{\text {nd }}$ Edition
}

\author{
Usbaldo Fraire, Jr. ${ }^{1}$ \\ Jacobs Engineering, Houston, TX, 77058 \\ Keith Anderson ${ }^{2}$ \\ ATK, Brigham City, Utah 84302 \\ Jose G. Varela ${ }^{3}$ \\ GeoControl Systems, Inc., Houston, TX, 77058 \\ Michael A. Bernatovich ${ }^{4}$ \\ NASA-Johnson Space Center, Houston, TX, 77058
}

\begin{abstract}
NASA's Orion Capsule Parachute Assembly System (CPAS) project has advanced into the third generation of its parachute test campaign and requires technically comprehensive modeling capabilities to simulate multibody dynamics (MBD) of test articles released from a C-17. Safely extracting a 30,000 lbm mated test article from a $\mathrm{C}-17$ and performing stable mid-air separation maneuvers requires an understanding of the interaction between elements in the test configuration and how they are influenced by extraction parachute performance, aircraft dynamics, aerodynamics, separation dynamics, and kinetic energy experienced by the system. During the real-time extraction and deployment sequences, these influences can be highly unsteady and difficult to bound. An avionics logic window based on time, pitch, and pitch rate is used to account for these effects and target a favorable separation state in real time. The Adams simulation has been employed to fine-tune this window, as well as predict and reconstruct the coupled dynamics of the Parachute Test Vehicle (PTV) and Cradle Platform Separation System (CPSS) from aircraft extraction through the mid-air separation event. The test-technique for the extraction of CPAS test articles has evolved with increased complexity and requires new modeling concepts to ensure the test article is delivered to a stable test condition for the programmer phase. Prompted by unexpected dynamics and hardware malfunctions in drop tests, these modeling improvements provide a more accurate loads prediction by incorporating a spring-damper line-model derived from the material properties. The qualification phase of CPAS testing is on the horizon and modeling increasingly complex test-techniques with Adams is vital to successfully qualify the Orion parachute system for human spaceflight.
\end{abstract}

\footnotetext{
${ }^{1}$ Aerospace Engineer, Aeroscience and Flight Mechanics, 2224 Bay Area Blvd, Houston, TX, AIAA Member.

${ }^{2}$ Aerospace Engineer, Advanced Engineering, P.O. Box 707, Brigham City, UT.

${ }^{3}$ Aerospace Engineer, Aeroscience and Flight Mechanics, 2224 Bay Area Blvd, Houston, TX, AIAA Sr. Member.

${ }^{4}$ Aerospace Engineer, Aeroscience and Flight Mechanics Division, Houston, TX.
}

American Institute of Aeronautics and Astronautics 


\section{Nomenclature}

\begin{tabular}{|c|c|c|}
\hline Aref & $=$ & Reference Area \\
\hline Adams & $=$ & Automatic Dynamic Analysis of Mechanical Systems \\
\hline BET & $=$ & Best Estimate Trajectory \\
\hline $\mathrm{C}_{\mathrm{a}}$ & $=$ & Axial force \\
\hline $\mathrm{C}_{\mathrm{N}}$ & $=$ & Normal force \\
\hline $\mathrm{CY}_{\mathrm{Y}}$ & $=$ & Side Force \\
\hline $\mathrm{C}_{11}$ & $=$ & Roll Moment \\
\hline $\mathrm{C}_{\mathrm{m}}$ & $=$ & Pitch Moment \\
\hline $\mathrm{C}_{\ln }$ & $=$ & Yaw Moment \\
\hline$C_{D} S_{r e f}(t)$ & $=$ & Extraction parachute apparent drag area as a function of time \\
\hline $\mathrm{C}_{\mathrm{x}}$ & $=$ & Aerodynamic Force coefficient \\
\hline $\mathrm{C}_{\mathrm{xx}}$ & $=$ & Aerodynamic Moment coefficient \\
\hline$c_{m}$ & $=$ & Center of Mass \\
\hline$c_{p}$ & $=$ & Center of Pressure \\
\hline CPSS & $=$ & Cradle Platform Separation System \\
\hline CPAS & $=$ & Capsule Parachute Assembly System \\
\hline$c_{r}$ & $=$ & Rotational Damping \\
\hline CG & $=$ & Center of Gravity \\
\hline DOF & $=$ & Degree of Freedom \\
\hline downdraft & $=$ & Downward z-component force applied on the composite extraction parachute \\
\hline EFTC & $=$ & Extraction Force Transfer Coupler \\
\hline EFTA & $=$ & Extraction Force Transfer Actuator \\
\hline EPJD & $=$ & Extraction Parachute Jettison Device \\
\hline Force $a e r o$ & $=$ & $Q \times S_{\text {ref }} \times C_{x}$ \\
\hline $\mathrm{k}$ & $=$ & stiffness coefficient \\
\hline KCm_ptv & $=$ & multiplier used to match PTV dynamics \\
\hline $\mathrm{L}_{\mathrm{ref}}-\mathrm{r}$ & $=$ & Reference Length \\
\hline Momentaero & $=$ & $Q \times S_{\text {ref }} \times L_{\text {ref }} \times C_{x x}$ \\
\hline Mated_cmq & $=$ & pitch damping outside aircraft \\
\hline Mated_cmq_wake & $=$ & pitch damping inside the wake \\
\hline MARM & $=$ & Mid-Air Release Mechanism \\
\hline Mated Vehicle & $=$ & PTV and CPSS \\
\hline MSL & $=$ & Mean Sea Level \\
\hline MRC & $=$ & Moment Reference Center \\
\hline PTV & $=$ & Parachute Test Vehicle ( 2 is the model name) \\
\hline $\mathrm{P}(\delta, \mathrm{n})$ & $=$ & Non-linear force function \\
\hline$\rho$ & $=$ & Atmospheric density \\
\hline $\bar{q}$ & $=$ & Dynamic Pressure $=1 / 2 \rho V^{2}$ \\
\hline QD & $=$ & Ramping function used to apply full aerodynamic forces as a function of wake length \\
\hline Ramp Pitch Angle & $=$ & C-17 Deck Angle \\
\hline Ramp Pitch Rate & $=$ & C-17 Deck Pitch Rate \\
\hline Sref & $=$ & Reference Area \\
\hline$v_{z} / v_{m}$ & $=$ & Normalized extraction parachute velocity \\
\hline
\end{tabular}




\section{Introduction}

Simulating CPAS extraction-separation testing techniques and assessing system performance requires an insight into multi-body dynamics, material properties, component mass properties, parachute performance and internal friction and external forces acting between objects interacting in the defined operating environments.

The objective of the first edition of this paper was to bound the separation timing and attitude dynamics of the mated test vehicle during the extraction-separation phase of flight using six primary modeling components: PTV, CPSS, Ramp, extraction parachute canopy, massless extraction line and a massless 31-ft programmer parachute deployment line used to sense the end of the simulation. The general motion of the mated vehicle as it exits the aircraft is of a single pendulum motion, Figure 1. The extraction parachute drag is the action force in the system that drives the pendulum motion. The extraction line is a single massless component force that is the reaction force that responds to the applied drag force and connects at the extraction parachute suspension line confluence point and

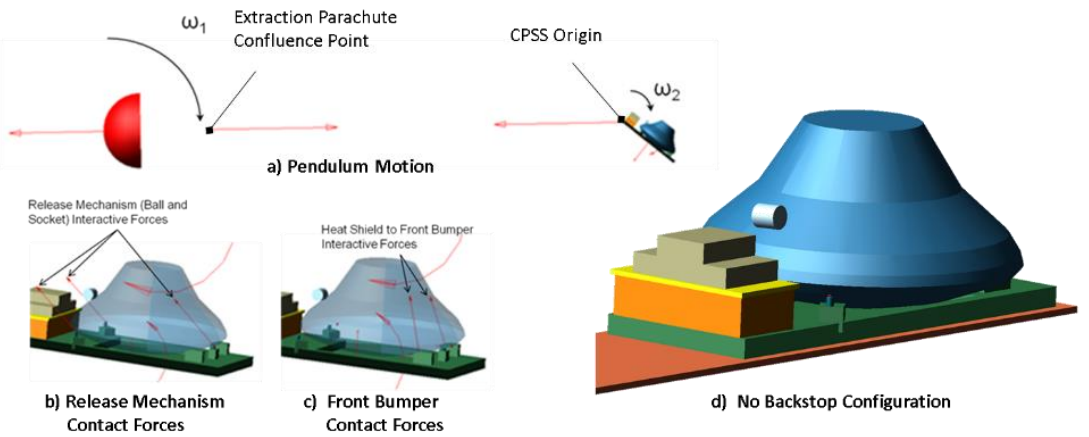

Figure 1: First Edition Adams Modeling Components extends to the CPSS origin, located at the aft, bottom, center of the platform. Contact forces between the CPSS/Ramp, PTV/CPSS release mechanism and PTV/CPSS front-bumpers were introduced to help tune aircraft exit velocities and separation timing events during post-test reconstructions. Each were anchored to the first three Engineering Development Unit (EDU) capsule tests. This extraction test technique uses an Extraction Force Transfer Coupling (EFTC) latch assembly attached on the centerline of the CPSS at the base of its aft end. The executed test technique relies on an aerodynamic based time varying extraction parachute pull force applied on the EFTC from the initial release of the mated vehicle along the ramp through the PTV/CPSS separation event. The EFTC is then released with a timed cutter when the CPSS has reached a defined altitude at which point it deploys the CPSS recovery system well after the PTV has been released. No high energy load transfers occur during the extraction-separation phase of flight.

The analysis demands of CPAS has evolved and progressively increased complexity with the introduction of a new backstop-reposition test technique required to mitigate unexpected dynamics and improve operational performance during the extraction event. The new backstop-reposition test technique involves a mid-air high energy or dynamic load transfer from a $24 \mathrm{lbm}$ Extraction Parachute Jettison Device (EPJD) to a 40-75 lbm CPAS confluence. The load transfer is activated at the instant an Extraction Force Transfer Actuator (EFTA) attached to the portside forward end of the CPSS platform clears the end of aircraft ramp. The EPJD is a critical device that connects three elements. The first element is the EFTC latch assembly that is attached to the CPSS platform edge. The second is the 140-ft Nylon Extraction Line and the third pin on the EPJD is designated for a 3-ft Kevlar Reposition Deployment Line which is routed to a CPAS confluence resting on the CPSS shelf. The confluence is then rigged with 8-ply Kevlar slings that connect to the four corners of the backstop. The Adams modeling configuration used to match the CPAS test configuration rigging route and the focus on the $2^{\text {nd }}$ edition is shown on Figure 2.

The extraction phase is comprised

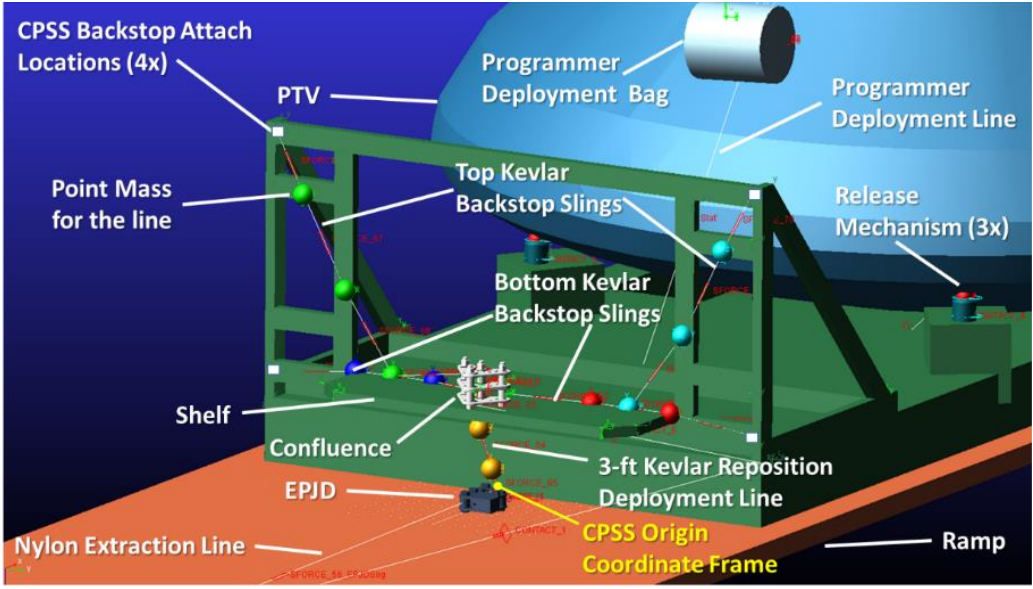

Figure 2: Second Edition Adams Backstop-Reposition Model of five critical sequential events. During the first extraction sequence, the entire extraction parachute pull force is applied on the connected EFTC latch 
assembly and EPJD located on the CPSS platform. As the platform reaches the end of the aircraft ramp the EFTA is activated and releases the EPJD. The Extraction Line continues to apply the pull force and when the EPJD travels the distance of the Kevlar Reposition Deployment Line it becomes taut and dynamically applies an initial load to the confluence which is released from the shelf. The confluence is now integrated into the load transfer path and accelerates until it travels the distance of the bottom slings at which point it suddenly decelerates momentarily with respect to the CPSS and reaccelerates until the entire system reaches an equilibrium state. The pendulum motion required to successfully release the PTV heat-shield forward is maintained by increasing the length of the backstop top slings to $12-\mathrm{ft}$ to ensure they do not become taut during this phase of flight. The additional masses introduced into the extraction parachute system are contributors to the overall system dynamics.

Modeling this dynamic high energy event in Adams requires an introduction of a new method for simulating the performance of line segments in the extraction parachute system. This new method is comprised of a series of pointmass connected by spring-damper forces as shown in Figure 3. The previous modeling method used one massless

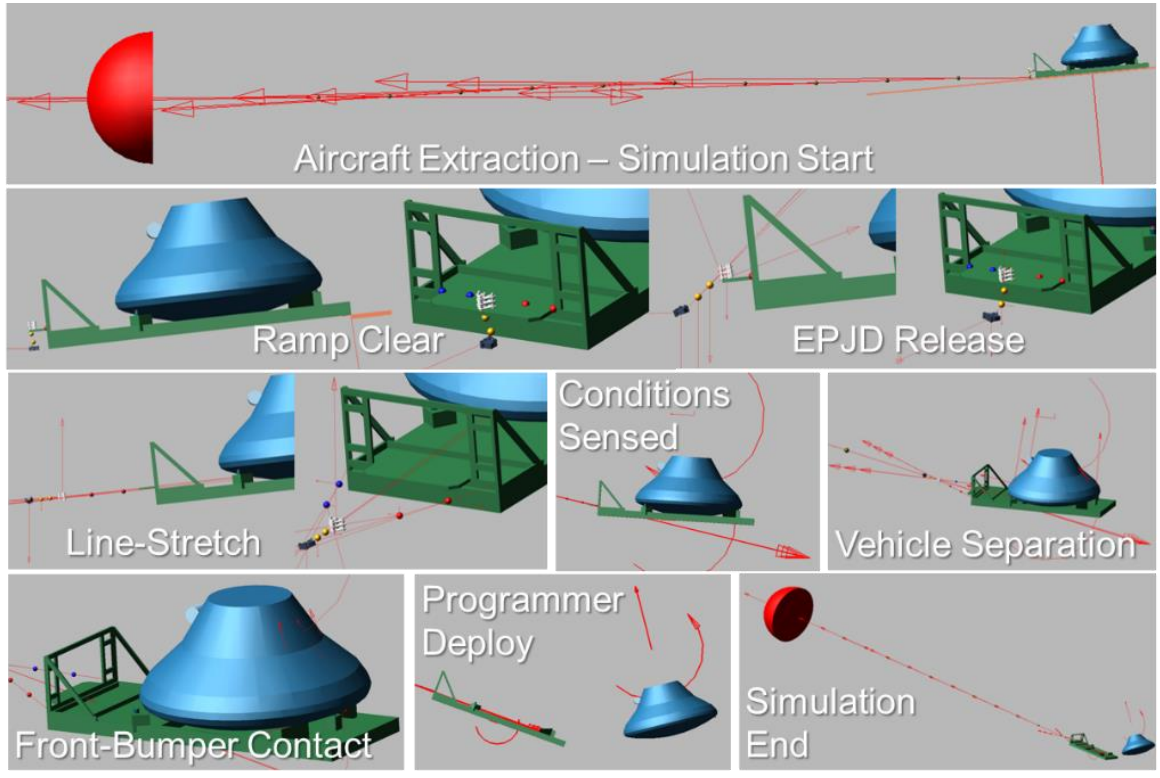

Figure 3: Adams End-to-End Simulation Sequence line element and did not capture the dynamics of the extraction line. As a result, the loads within the extraction line and transferring into the EPJD was not accurately simulated. The dynamic peak loads resulted in negative margins for line segments and hardware components used in the current CPAS test configuration. The introduction of serial pointmasses has helped decelerate the systems deployment sequence and is being refined to produce representative dynamic peak loads to reduce the probability of line segment and hardware malfunctions. The spring-damper forces are adjusted based on material properties. Kevlar and Nylon are the primary materials used in the test configuration. Textile performance is based on material properties and allow the lines to elongate using derived linear stiffness coefficients as inputs.

Simulating the integrated system begins by constraining each geometry and point mass using various primitive joints in Adams to control fixed, translational, orientation and in-plane motions. This modeling approach controls the timing of the deployment sequence. A simulation control logic script executes a series of commands that allows the user to release constraints or deploy objects in a specified order when defined sensors have been satisfied. As the simulation is executing, the elements in the model act and react with respect to each other based on the energy or drag force in the system. Linear viscous and rotational damping terms are applied to remove numerical instability in the solution caused by unrealistic oscillations in the lines, over-rotation of geometries that produce excessive twisting in the lines such as the confluence, connectors and point masses.

The new CPAS extraction technique has introduced dynamic loads into the system that must be understood to analyze textile and hardware performance limits to safeguard against mid-air malfunctions. The first edition of this paper only focused on dynamics. Adams modeling has evolved to meet the need for loads predictions required to ensure that upcoming CPAS test configurations will survive the experienced testing environments. Hardware malfunctions have been observed during two EDU tests. From an operational perspective, the malfunction was attributed to the applied build-up confluence rigging configuration that failed due to the unexpected kinetic energy experienced during the initial extraction deployment sequence. The analytical perspective arrived at a conclusion that there was a deficiency in modeling and understanding of confluence dynamics at the tested environments. The flight test resulted in the confluence experiencing loads exceeding the strength of a Kevlar cut cord, rated to $1400 \mathrm{lbf}$, used in the confluence rigging process. The consequence was a premature deployment of the CPSS Recovery Mains at $13,000-\mathrm{ft}$ and 35,000-ft. 
The current modeling developments have been accelerated to improve the understanding of confluence dynamics and the internal force interactions between objects deployed at 35,000 ft. The current state of the simulation shown in Figure 3 illustrates how point masses have been introduced to increase the fidelity of the line modeling. Geometries with mass properties for all the elements in the extraction parachute system have also been integrated into the current Adams model to produce representative dynamic load predictions. Material property characteristics for each line segment used in the system are modeled using derived spring stiffness and damping coefficients that define elongation which is used to determine line survivability during deployment sequences. Integrating all the elements described improves the fidelity of loads and dynamics predictions. Adams modeling improvements have been implemented to simulate reality and validate the performance of a test technique required to support a successful CPAS test campaign.

\section{Modeling Aircraft Dynamics at Extraction}

Modeling aircraft dynamics during the extraction phase requires analysis of pitch plane test data to gain an understanding of the possible test vehicle responses as it reaches the end of ramp. The first two PTV tests used heritage data and defined conservative dispersion ranges for the pitch rate $\left(0 \%\right.$ to $\left.5.33^{\circ} / \mathrm{s}\right)$ and ramp angle $\left(0^{\circ}\right.$ to $\left.5^{\circ}\right)$ to bound possible aircraft dynamics scenarios and used for Monte Carlo assessments ${ }^{4}$. As the CPAS EDU test campaign progressed, the availability of test data increased and contributed to the refinement of aircraft ramp modeling during extraction.

In the Adams simulation, the aircraft dynamics are modeled using a ramp which is
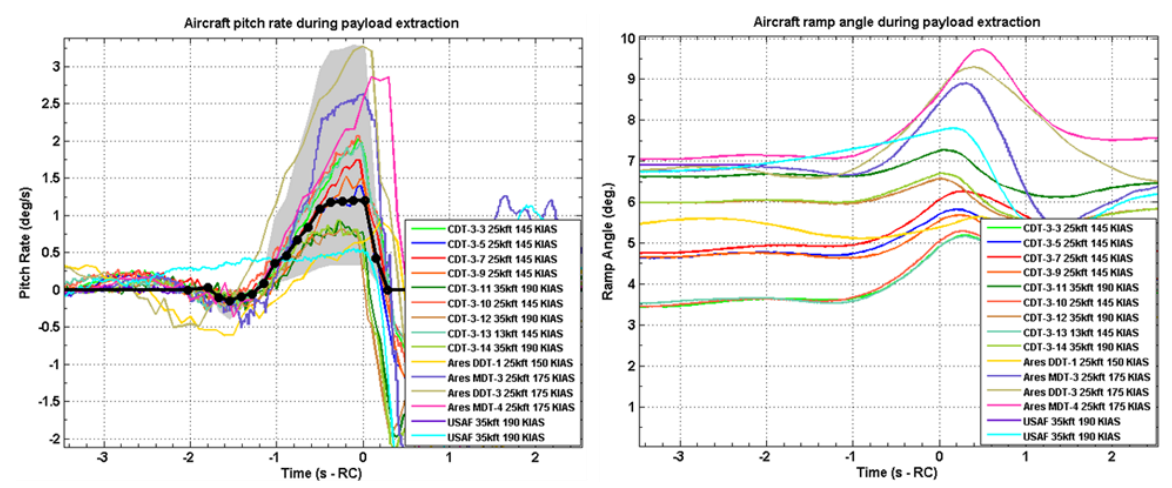

Figure 4: Carrier aircraft pitch rate (left) and deck angle response (right) to various C-17 payload extractions initialized with a user defined pitch angle that remains constant and pitch rate profile that varies as the vehicle approaches ramp clear. The updated ranges for the aircraft deck angle are $3.5^{\circ}$ to $5^{\circ}$ for extractions at $25,000 \mathrm{ft}-\mathrm{MSL}$ and $5^{\circ}$ to $7^{\circ}$ at $35,000 \mathrm{ft}-\mathrm{MSL}$. Figure 4 shows carrier aircraft pitch rate and deck angle profiles for various drops tests conducted by CPAS, Ares and the USAF relative to ramp clear. Each pitch rate profile represents the aircraft reaction to a designed $1 \mathrm{G}$ payload extraction, with the exception of the cyan trace which was an intentional $0.5 \mathrm{G}$ extraction. This data set has been used to adjust the dispersion factor identified as the gray shaded area in the plot. The coefficient range is applied to the mean pitch rate data set, which in Figure 4 is the solid dotted black line, aiding in pre-flight analysis.

Using the ramp pitch rate profile in concert with an initial ramp angle has added modeling fidelity to this phase of flight in the simulation. Varying the ramp angle and rate introduces variation in the final state vector of the

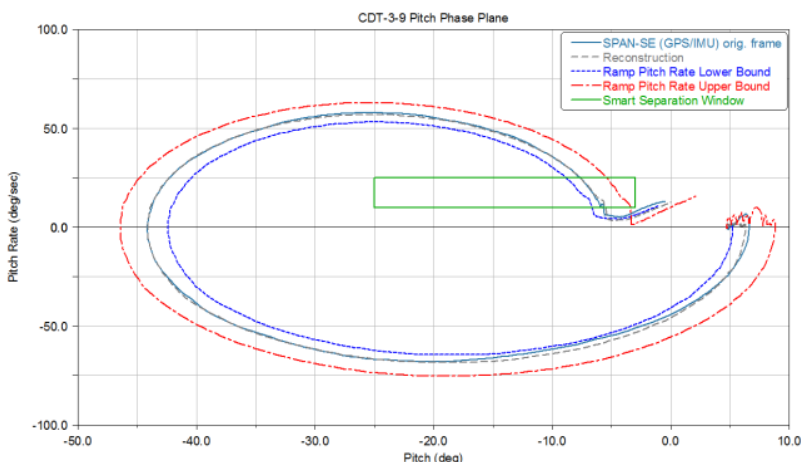

Figure 5: Aircraft Tip-off Motion Effects on PTV/CPSS Dynamics

American Institute of Aeronautics and Astronautics mated vehicle at ramp clear that carry through the end of the simulation. This perturbance becomes evident when looking at the EDU-A-CDT-3-9 reconstruction summary on Figure 5. Matching the initial tip-off conditions at ramp clear support correlating the initial downward pitch motion. If the pitch rate profile is shifted to a lower or upper bound, the response of the mated vehicle dynamics are apparent. The upper bound (red) produces a wider trajectory and enters closer towards the right edge of the logic window. The lower bound (blue) produces the opposite effect. The trajectory is more compressed and enters closer to the center of the logic window. Overall, the additional test data has supported defining a representative range of aircraft pitch angle and pitch rate profiles. 


\section{Aerodynamics}

The aerodynamic forces experienced during free-flight are critical to understanding the behavior of objects in a free stream air flow as independent bodies and while in close proximity to other objects. The following are the fundamental equations that were used to account for the 18 aerodynamic effects considered in the simulation for a mated vehicle, PTV alone and CPSS alone.

\section{A. Applied Vehicle Aerodynamic Forces-Moments in Free-Flight}

Six aerodynamic force components are used to define the total external aerodynamic force acting on a body as it free-falls through the atmosphere. The form of these force components are described by two equations.

$$
\begin{gathered}
F_{\text {aero }}=\bar{q} C_{x} S_{\text {ref }}=1 / 2 \rho V^{2} C_{x}(\alpha, \beta) S_{\text {ref }} \\
M_{\text {aero }}=\bar{q} C_{x x} S_{\text {ref }} L_{\text {ref }}=1 / 2 \rho V^{2} C_{x x}(\alpha, \beta) S_{\text {ref }} L_{r e f}
\end{gathered}
$$

The variable $\bar{q}$ is the dynamic pressure, $\mathrm{S}_{\mathrm{ref}}$ is the reference area, $\mathrm{L}_{\mathrm{ref}}$ is the reference length, and $\mathrm{C}_{\mathrm{x}}, \mathrm{C}_{\mathrm{xx}}$ are the respective aerodynamic coefficients associated with each degree of freedom (DOF). The coordinate systems and reference parameters used to define the mated body PTV2+CPSS, CPSS pallet system alone and PTV2 capsule alone aerodynamic coefficients is shown in Figure 6. The aerodynamic forces include components for axial, $\mathrm{C}_{\mathrm{a}}$, normal, $\mathrm{C}_{\mathrm{N}}$, and side, $\mathrm{C}_{\mathrm{Y}}$. The aerodynamic moment components are for roll, $\mathrm{C}_{\mathrm{ll}}$, pitch, $\mathrm{C}_{\mathrm{m}}$ and yaw, $\mathrm{C}_{\mathrm{ln}}$.
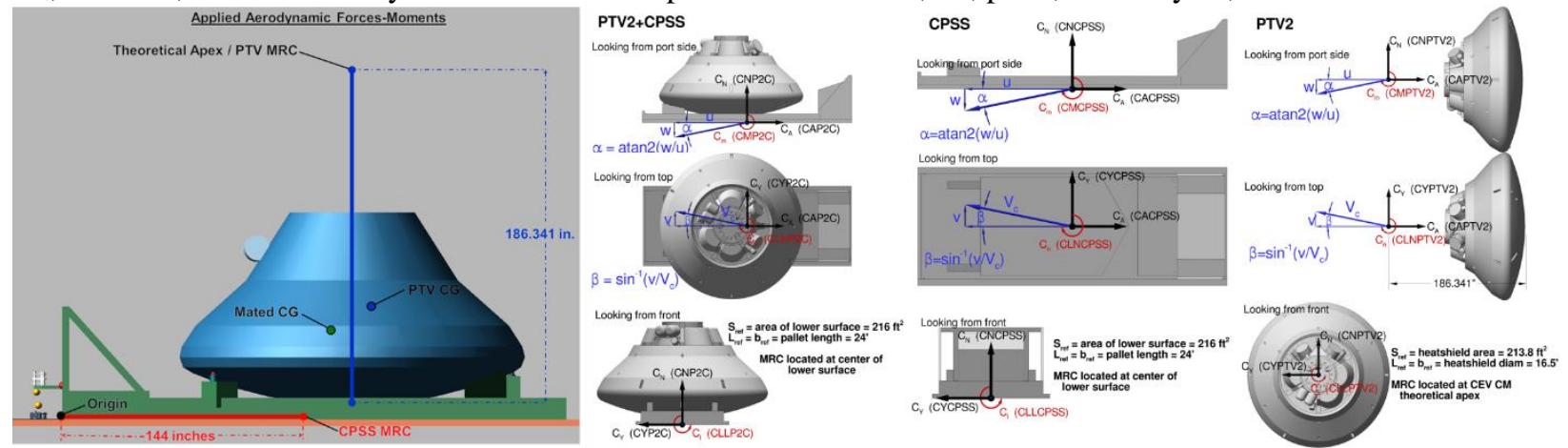

Figure 6: Simulation and Aerodynamic Database Version 3.2.0 Definitions and Coordinate System ${ }^{5}$

The aerodynamic database that is applied in the Adams simulation is provided by the CPAS project and was derived from wind tunnel data and computational fluid dynamics (CFD) analysis ${ }^{5}$. The Moment Reference Center (MRC) for each of these datasets in the simulation are applied at the PTV theoretical apex (186.341 inches from the bottom of the heat shield) and at the center of the CPSS pallet (144 inches from the Origin).

\section{B. Applied Aerodynamic Forces-Moments in Close Aircraft Proximity}

Prior to extraction parachute inflation, the mated vehicle is restrained on the $\mathrm{C}-17 \mathrm{ramp}$ and no aerodynamic forces act on the bodies. As inflation process progresses, the canopy drag force is applied at the CPSS Origin and the vehicle begins its translational motion down the ramp as shown in Figure 7.

The vehicle continues to travel along the ramp until its forward edge has cleared the ramp. At ramp clear, the mated vehicle is in close proximity to the aircraft and a ramping function applies aerodynamic forces to the bodies according to the following relationship, QD.

$$
Q D=\left[\frac{1-\left(D_{\text {wake }}-d_{\text {ramp }}\right)}{D_{\text {wake }}}\right] * F_{\text {aero }}
$$

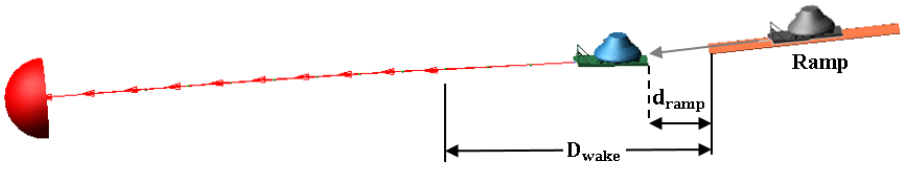

Figure 7: Aero Force Diagram While In Close Proximity to the Aircraft

The ramp distance, $d_{\text {ramp }}$, is measured by two markers. One is located at the aft edge of the ramp and a second marker is at the forward edge of the CPSS. The distance is negative while on ramp, zero at ramp clear and positive after the vehicle exits the aircraft. The mated aerodynamic forces take full effect when the separation distance exceeds the wake length, $D_{\text {wake }}$, and continue to apply until the vehicle reaches the separation conditions. Derived wake length magnitudes vary from 83 -ft to $100-\mathrm{ft}$. At PTV2 and CPSS separation, the mated vehicle aerodynamic forces are deactivated and the independent body aerodynamic coefficients are activated instantaneously for the remainder of the simulation run.

American Institute of Aeronautics and Astronautics 


\section{Reconstruction Process Using Aerodynamic Parameters to Correlate Dynamics}

Following each test, a post test reconstruction is made in ADAMS to the actual flight test data to establish the dispersion of the nominal parameters to the "Best Fit" values. The general process used to reconstruction each test includes the following steps.

1. Input the actual initial altitude, velocity, mass, atmospheric properties and ramp deck angle into the simulation.

2. Adjust the Extraction Parachute apparent drag area, $C_{d} S$, the inflation parameters and beginning of inflation sequence to match the performance of the mated PTV2/CPSS while on the aircraft ramp.

3. Adjust the length of the distance, $D_{\text {wake }}$, which is considered to be distance the mated bodies are in the wake of the aircraft. Also adjust any other applicable aerodynamic coefficients so that the magnitude of the pitch oscillation and period of oscillation match test data.

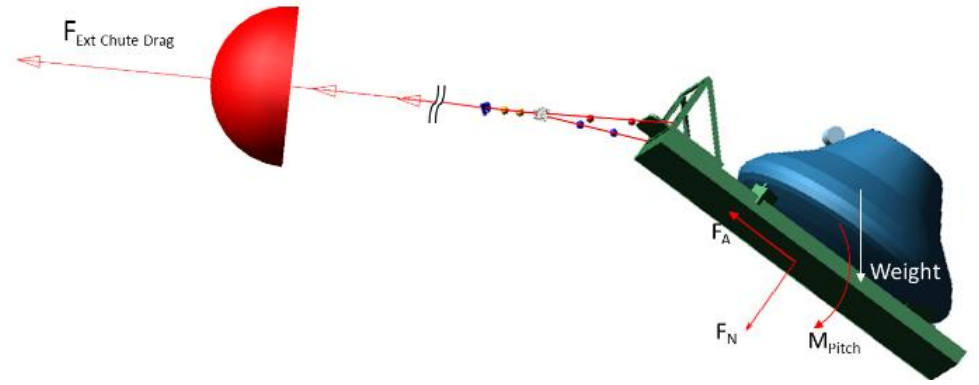

Figure 8: CPAS Drop Test Overall System Free Body Diagram
In order to get a good match to the actual test data, sensitivity analysis was performed to determine the parameters that are most significant to matching the motion of the mated CPSS/PTV2 under the extraction parachutes. Newton's second law of motion is used to describe the fundamental equations that govern the general motion of the mated CPSS/PTV2 during extraction and separation phase of flight. A free body diagram for this phase of flight is shown in Figure 8.

The driving forces experienced by the system are the extraction parachute drag force, $F_{\text {Ext Chute Drag }}$, axial force, $F_{A}$, normal force, $F_{N}$, and weight, W. Summing the forces gives the following relationship.

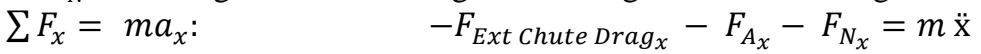

$$
\begin{aligned}
& \sum F_{y}=m a_{y}: \quad 0=m \ddot{\mathrm{x}}
\end{aligned}
$$

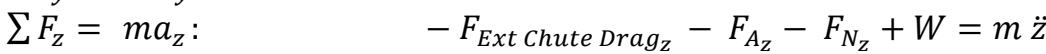

In the Equations 4-6, the weight, mass and motion of the system are measured entities. The unknowns include the aerodynamic forces. The aerodynamic forces are defined by Equations 1 and 2 . The dynamic pressure, $\bar{q}$, is calculated using day of flight weather balloon air density data. The initial velocity of the system is acquired using an onboard avionics tray. The employed C-17 typically maintains a constant velocity and altitude, therefore, initial velocity conditions can be derived by $\mathrm{Vel}_{\mathrm{x}}=\mathrm{Vel}_{\mathrm{C}-17}$ and $\mathrm{Vel}_{\mathrm{z}}=0$. The remaining unknowns in Equations 1 and 2 are the aerodynamic coefficients of the CPSS/PTV2 mated body and the extraction parachute apparent drag area, $\mathrm{C}_{\mathrm{d}} \mathrm{S}$, which is the product of the drag coefficient and drag area. It should be noted that while the CPSS/PTV2 is in the aircraft, the aerodynamic forces on the CPSS/PTV2 are zero and that leaves the only unknown in Equations $1 \& 2$ of $\mathrm{C}_{\mathrm{d}} \mathrm{S}$. If the sequence of events while on the ramp, such as extraction parachute inflation, first motion, leading edge of CPSS off the ramp and trailing edge off the ramp, is matched then we have a pretty good grasp of the $C_{d} S$ term for the extraction parachute. Since the extraction parachute is fully opened and inflated by the end of ramp, then $\mathrm{C}_{\mathrm{d}} \mathrm{S}$ will remain a constant for the rest of the extraction/separation phase of testing. Once the $\mathrm{C}_{\mathrm{d}} \mathrm{S}$ value is adjusted to get a good correlation of the ramp events, the second step in the reconstruction process is completed. After the completion of Step 2, a good correlation exists for the overall trajectory of the test hardware during the extraction/separation phase of testing. The PTV2 X \& Z direction velocities correlate well to the test data. This is true because the extraction parachute drag force is an order of magnitude greater than any other aerodynamic force acting on the bodies and the weight is a measured value with a good degree of confidence in

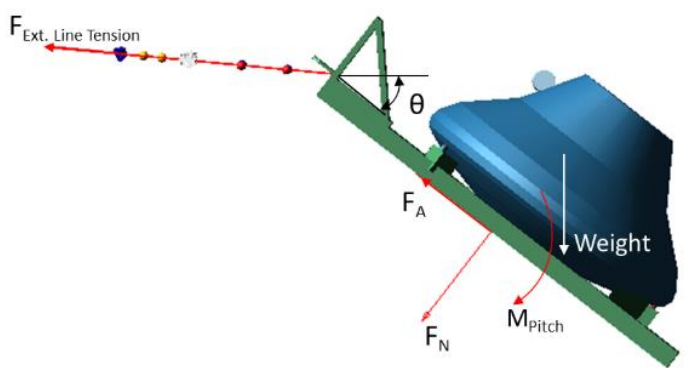

Figure 9: Mated Body Free Body Diagram its accuracy. 
The final step in the standard reconstruction process of a CPAS drop test becomes the correlation of the pitch and pitch rate data. The governing equation for this correlation is derived by summing moments about the mated vehicle cg as shown in Figure 9 and yields an equation of the form,

$$
\sum M_{\text {pitch }}=I_{y y} \ddot{\theta}=F_{\text {Ext.Line Tension }} l_{1}-F_{A} l_{2}+F_{N} l_{3}-M_{\text {pitch }} \text { (7) }
$$

The known terms and components in Equation 7 are the lever arms derived from test geometry, the mass properties $\left(\mathrm{I}_{\mathrm{yy}}\right)$, extraction line tension and $\ddot{\Theta}$ ( $\ddot{\Theta}$ can be calculated by taking the derivative of the pitch rate). The unknown terms are the aerodynamic forces and the line of action the extraction line force makes with respect to the CPSS. This line of action drives the length of the lever arm $\left(l_{1}\right)$ the extraction line tension vector makes with respect to the mated body cg.

Analysis was performed to understand the sensitivity of the pitch plane swing to line of action of the

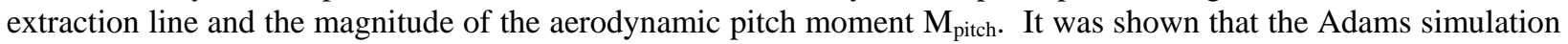
could be tuned to actual test data by adjusting either the line of action of the extraction line force vector or the pitch moment. The line of action the extraction line force pulls on the CPSS could vary from test to test due to wind turbulence in the wake of the aircraft acting on the extraction chutes, wind gusts in random directions, orientation of the extraction chutes relative one to another. In this paper, we will focus on adjustments made to the pitch plane aerodynamic moment.

The pitch plane aerodynamic moment is calculated from two coefficients, the pitching moment coefficient, $\mathrm{C}_{\mathrm{m}}$, and a pitching moment damping component defined by a $\mathrm{C}_{\mathrm{mq}}$ term. The total pitch moment is defined as,

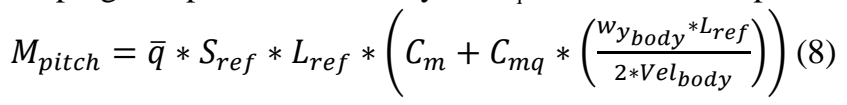

Probably the least understood parameter in this equation is the $\mathrm{C}_{\mathrm{mq}}$ term and this is the parameter we chose to adjust to correlate the simulation to actual data. To get the best fit of the actual data, it was decided to use two $\mathrm{C}_{\mathrm{mq}}$ terms, one while the mated bodies are in the wake of the aircraft and one when the mated bodies are in the far field steady state airflow. Factors that could contribute variation of the $\mathrm{C}_{\mathrm{mq}}$ term is the turbulence of the airflow while in the wake, the length of the wake and direction of the angular motion.

There have been seven drop test where no anomalies occurred which we can reconstruct the flight trajectory and then compare the variation of the wake and aerodynamic $\mathrm{C}_{\mathrm{mq}}$ terms. Table 1 summarizes the initial conditions and key inputs into the Adams simulation for each of the seven tests.

Table 1: CPAS Drop Test Initial Conditions and Inputs

\begin{tabular}{|l|l|l|l|l|l|}
\hline Test ID & Alt (K-ft) & Velocity (ft/s) & $\begin{array}{l}\text { Ramp Angle, } \\
\text { deg }\end{array}$ & Mass, lbm & Iyy, lbm in2 \\
\hline CDT33 & 25,249 & 360 & 3.6 & 31339 & $1.889 \mathrm{E} 8$ \\
\hline CDT35 & 25,079 & 359 & 4.7 & 31901 & $1.874 \mathrm{E} 8$ \\
\hline CDT37 & 24,990 & 353 & 4.9 & 32000 & $1.9 \mathrm{E} 8$ \\
\hline CDT39 & 24,988 & 371 & 4.75 & 32000 & $1.9 \mathrm{E} 8$ \\
\hline CDT310 & 25,041 & 360 & 3.7 & 34322 & $2.032 \mathrm{E} 8$ \\
\hline CDT311 & 35,264 & 555.7 & 7.50 & 32271 & $1.92 \mathrm{e}+08$ \\
\hline CDT315 & 35,264 & 555.7 & 3.7 & 34322 & $2.032 \mathrm{E} 8$ \\
\hline
\end{tabular}

Table 2 summarizes the parameters that were adjusted to get the best match of the Adams simulation to the actual data.

Table 2: CPAS Reconstructed Aerodynamic Properties

\begin{tabular}{|l|l|l|l|l|}
\hline Test ID & Mated_cmq_wake & Mated_cmq & $\begin{array}{l}\text { Wake Distance, D, } \\
\text { inches }\end{array}$ & KCm_ptv \\
\hline CDT33 & -0.30 & 5.9 & 1200 & 0.84 \\
\hline CDT35 & -3.80 & 9.6 & 1200 & 0.95 \\
\hline CDT37 & 5.50 & 0.2 & 1000 & 0.85 \\
\hline CDT39 & 5.60 & 0.5 & 1000 & 1.0 \\
\hline CDT310* & 4.00 & 0.2 & 1000 & 1.22 \\
\hline CDT311 & 3.5 & -0.3 & 1000 & 1.0 \\
\hline CDT315* & 4.0 & 0.2 & 1000 & 1.22 \\
\hline
\end{tabular}

*Only tests with a backstop reposition. All other tests are a standard load transfer test. 
The reconstructed pitch plane damping values for CDT-3-3 and CDT-3-5 are in a different range than the final three tests. The most notable difference in the configuration of the test was that the EPJD for CDT-3-3 and CDT-3-5 remained attached to the CPSS and did not reposition so the load path included the confluence and CPSS slings. When the configuration includes the reposition, the point of attach on the CPSS is raised by 15 inches and changes the lever arm of the extraction line force (See Equation 7 and Figure 9). The range of the Mated_Cmq_wake for configuration which included the reposition was from 4.0 to 5.6 and 0.2 to 0.5 when out of the wake. The other parameter that were adjusted during a reconstruction was a multiplier on the PTV alone pitching moment aerodynamic coefficient, KCm_PTV. By adjusting the KCm_PTV multiplier, a good correlation was achieved for the slope of the PTV pitch rate after separation.

\section{Modeling Line Performance Using Material Properties and a Finite Element Approach}

Understanding force interactions between elements in the CPAS system is vital to ensure performance capabilities are not exceeded during in-flight maneuvers. Adams was initially used exclusively to assess system attitude dynamics and evolved to account for finite element analysis of Orion test vehicle elements in the high energy deployment sequence at extraction. Early line modeling attempts used linear stiffness and damping coefficient derivations to produce loads predictions, but proved to be conservative and generated negative margins for hardware components and textile lines. Modeling elements where assumed to be massless-frictionless objects which contributed to faster deployment sequences than flight. The subsequent sections describes the evolution of assumptions, derived non-linear force functions and damping considerations.

\section{A. Stiffness Coefficient}

The general force expression used to model a mass-spring-damper system is described as the following.

$$
F_{\text {total }}=F_{s}+F_{d} \Rightarrow m \ddot{x}=-c \dot{x}-k x=0
$$

The first component accounts for the spring stiffness, $F_{s}$, where $\mathrm{k}$ is the linear stiffness coefficient and $\mathrm{x}$ is the displacement. The second component is the damping force, $F_{d}$, where $\mathrm{c}$ is the damping coefficient and $\dot{x}$ is the velocity.

\section{Linear Stiffness Coefficient Derivation}

For CPAS textiles, the linear stiffness coefficient and force function was derived using line specific material properties.

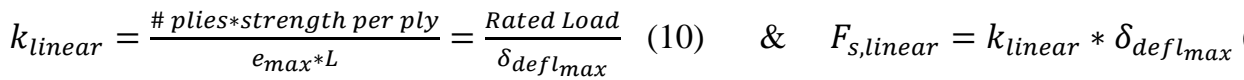

The numerator in Equation 10 is the rated load of the line and number of plies varies based on construction. The strength per ply for Nylon and Kevlar is $15,000 \mathrm{lbf}$ and 20,000 lbf, respectively. The material strain, $\mathrm{e}_{\max }$, used is the maximum displacement the line can stretch prior to failure. The maximum strain values used for Nylon and Kevlar are $2.5 \%$ and $20 \%$, which is assumed from test experience. Ground testing is planned to better understand the material performance of CPAS specific slings used in flight. The denominator represents the maximum deflection of the line, $\delta_{\text {defl }} l_{\max }$. The $c_{\text {linear }}$ is a function of $k_{\text {linear }}$ and $\mathrm{m}$ is the mass of the element system. Using Equations 10 and 11 to produce loads predictions proved too conservative and a novel derivation method was employed using a non-linear force function ${ }^{6}$

\section{Novel Non-Linear Stiffness Coefficient Derivation}

To alleviate conservative loads predictions a novel non-linear force function was introduced to soften the line during the initial dynamic deployment events that occur at time $<0.5 \mathrm{~s}$. The linear stiffness method assumes a failure strain at the instant the line experienced any force and resulted in analysis predicting hardware and textile safety margin exceedances. In reality, CPAS textiles are softer due to their weave construction and allow more stretching to occur during the transient high energy maneuvers. As a result, a non-linear approach was adopted and is based on an experimental observation that relates the kinetic energy, $E_{K E}$, in the system with the strain energy, $E_{S E}$, of a specific line that absorbs the kinetic energy ${ }^{6}$

$$
E_{S E}=E_{K E} \Rightarrow \frac{1}{2} k x^{2}=\frac{1}{2} m v^{2}
$$

The non-linear force function used to predict loads is derived using equation 12 and assuming the relationship between the load and displacement at failure.

$$
P_{\max }\left(\delta_{\text {defl } l_{\max }}\right)=A *\left(\delta_{\text {def } l_{\max }}\right)^{2}+B *\left(\delta_{\text {def } l_{\max }}\right)(13)
$$

The strain energy shown in Equation 14 is also expressed as a function of the displacement at failure and we now have two equations with $\mathrm{A}$ and $\mathrm{B}$ remaining as unknowns. The $\mathrm{n}$ value is an experimental curve-fit term and may be between 2 and 3 depending on the curvature of the force-displacement curve for a specific line.

American Institute of Aeronautics and Astronautics 


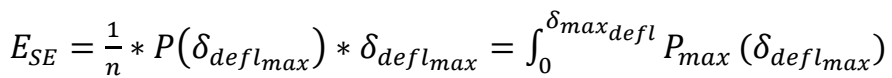

Solving for $\mathrm{B}$ in equation 13 and substituting into equation 14 after integrating, we arrive at the non-linear force function in terms of $\mathrm{n}$ and $\delta_{\text {deflmax }}$ used in the Adams simulation to produce loads.

$$
P(n, \delta)=A(n) * \delta^{2}+B(n) * \delta, \quad \text { where } \delta=0,0.1 \text { inch }, \ldots \delta_{\text {defl }} \text { max }
$$

A summary of the force-deflection curves used for CPAS specific lines are shown in the Figure 10. A conceptual example is included to depict the difference between a linear (red) and non-linear (blue) force function. As the fibers of the Nylon or Kevlar webbing are stretched and align the deflection of the material follow the blue curvature
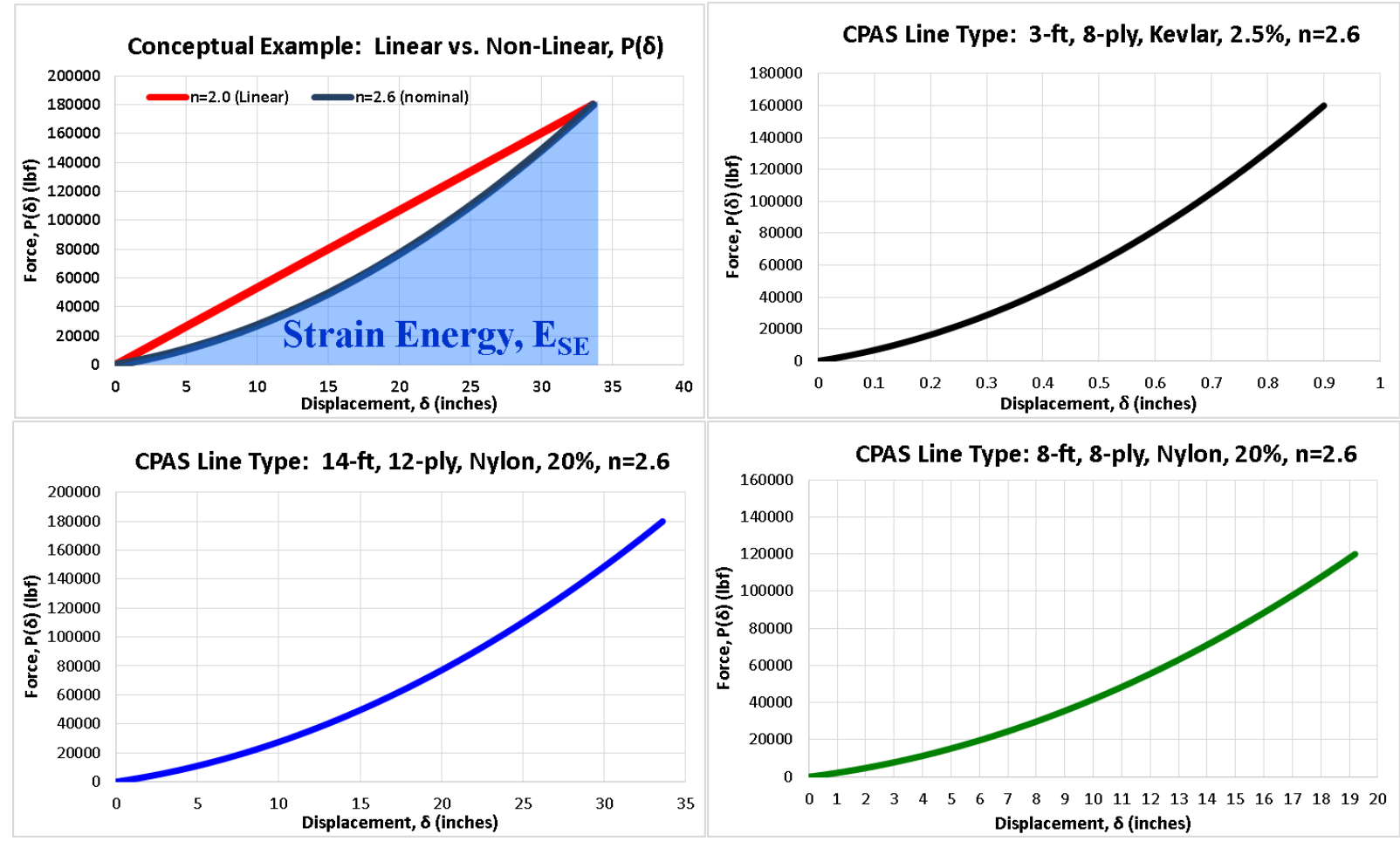

Figure 10: Non-linear force function

as shown on Figure 10. Each curve is defined by Equation 15 with the max load representing the failure point based on the material properties. The maximum operating loads conditions for each line is 30,000-lbf and 60,000-lbf for a $25,000-\mathrm{ft}$ and $35,000-\mathrm{ft}$ extraction, respectively. The area under the linear curve is of a triangle $(1 / 2 *$ base $*$ height $)$, where $n=2$. The non-linear curves each use the same fundamental area formula, except $n$ is set to 2.6 to curve-fit test data from comparable material samples.

The $A(n)$ and $B(n)$ terms are calculated for each line element and have units of lbf^2/in and lbf/in, respectively. Solving for the non-linear stiffness coefficient using equation (15) we arrive at the new non-linear spring stiffness coefficient function.

$$
k(n, \delta)=\frac{P(n, \delta)}{\delta}=A(n) * \delta+B(n)
$$

A summary of the derived material properties is shown in

\begin{tabular}{|c|c|c|c|c|c|c|c|c|c|c|}
\hline $\begin{array}{l}\text { Data } \\
\text { Source }\end{array}$ & $\begin{array}{c}\text { Material } \\
\text { (Type) }\end{array}$ & $\begin{array}{c}\text { Max Strain } \\
(\%)\end{array}$ & $\begin{array}{c}\text { No. Plies } \\
(\#)\end{array}$ & $\begin{array}{l}\text { Length } \\
\text { (inches) }\end{array}$ & $\begin{array}{c}\text { Deflection } \\
\text { (inches) }\end{array}$ & $\begin{array}{c}n \\
\text { (no units) }\end{array}$ & $\begin{array}{c}A(n) \\
\left(l b f / i^{A} 2\right)\end{array}$ & $\begin{array}{c}B(n) \\
(\mathrm{lbf} / \mathrm{in})\end{array}$ & $\begin{array}{l}\text { Stiffness Coefficient } \\
\text { (lbf/in) }\end{array}$ & $\begin{array}{l}\text { Peak Load } \\
\quad \text { (lbf) }\end{array}$ \\
\hline Accel. (2000g) & Kevlar & 2.5 & 8 & 36 & \begin{tabular}{|l|}
0.35 \\
\end{tabular} & 2.6 & 136752 & 54701 & 102564 & 36375 \\
\hline SEF PIN (STBD) & Nylon & 20 & 8 & 96 & 8.64 & 2.6 & 225 & 1923 & 3870 & 33421 \\
\hline SEF PIN (PORT) & Nylon & 20 & 8 & 96 & 8.59 & 2.6 & 225 & 1923 & 3858 & 33133 \\
\hline
\end{tabular}

Table 3.

Table 3: CPAS Specific Line Material Properties

American Institute of Aeronautics and Astronautics 


\section{B. Damping Coefficient}

Three types of damping terms are used in the Adams simulation to help stabilize the lines, discrete masses and confluences used by the extraction system during the deployment sequences. Damping values are required in the mass-spring-damper function to help calculate finite element load predictions for each line. Linear viscous damping is applied to discrete point masses to stabilize the masses similar to flight. The third damping type is rotational damping and is applied to confluences in the system like the EPJD, MARM or 6:2 Confluence to prevent unrealistic twisting in the lines caused by over-rotation of the hardware. Introducing damping into a simulation helps settle object dynamics and allows for smoother execution run times. When introducing damping careful considerations must be invested into verifying that the system being analyzed does not end up over-damped. An over-damped simulation impacts loads and dynamics results.

1. Deriving Damping Coefficients for Specific CPAS Lines

The second half of equation 9 for predicting loads is the damping coefficient. The general process for deriving a damping coefficient begins with understanding the natural frequency of the system, event sequencing and

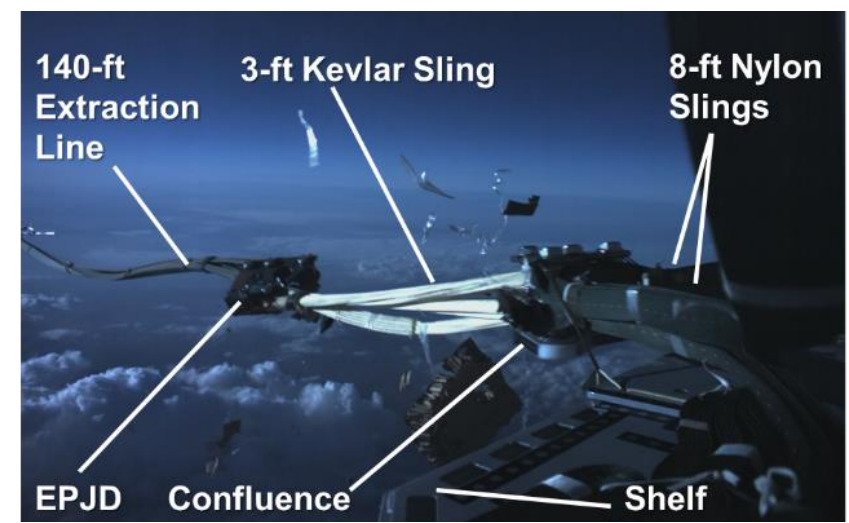

Figure 11: High Speed Backstop Reposition Event specifications of the sensors used to acquire flight data. The first step is to calculate the natural frequency (Eq. 17) of the line being analyzed. In this case, the line is a 3 -ft Kevlar sling.

$$
f_{n}=\frac{1}{2 \pi} \sqrt{k / m}
$$

The $\mathrm{k}$ is calculated using Equation 16 and $\mathrm{m}$ is the mass of the object connected by the line. In this case, $\mathrm{m}$ is a $43 \mathrm{lbm}$ confluence connected to a $1.5 \mathrm{lbm}$ Kevlar sling. A snapshot of the dynamic event being analyzed is shown in the Figure 11.

The natural frequency is used as an independent check to isolate peak loads that correspond to the line being analyzed and separate false peak loads attributed to other external influences due to the systems' deployment sequence (i.e. Nylon extraction line and slings, $24 \mathrm{lbm}$ EPJD). Not understanding the natural frequency can introduce uncertainty in the final results if the corresponding peak amplitudes are not selected.

A conceptual example along with the analyzed 6:2 accelerometer data is shown in Figure 12. The peak amplitudes are selected and checked based on the natural frequency of the line to ensure the best data set is included in the derivation. As seen at time $15.195 \mathrm{~s}$, the peak amplitude was not selected, because it did not occur at the derived natural frequency and may likely be attributed to a rotational motion between the EPJD and 6:2 confluence.
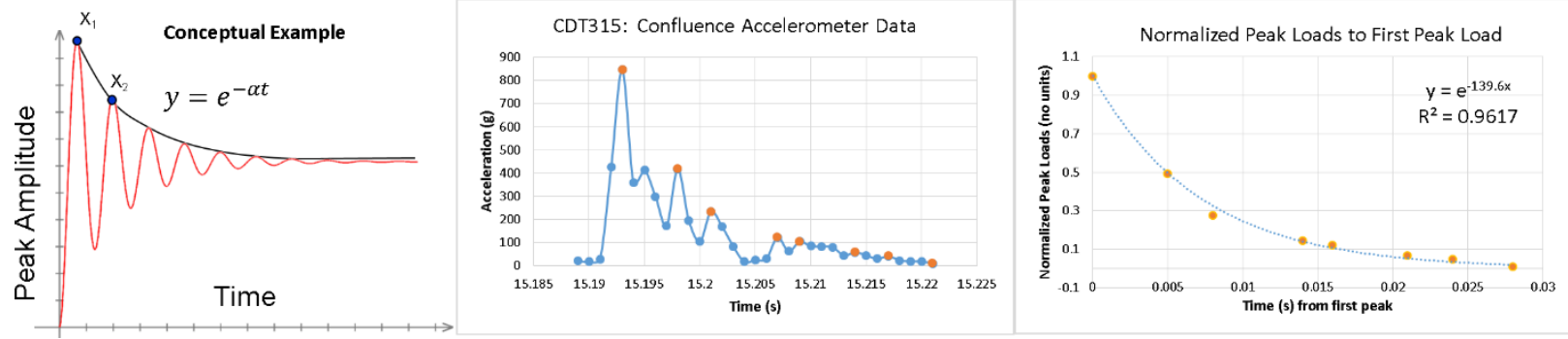

Figure 12: EDU-A-CDT-3-15 Accelerometer Data

Eight data points were selected for this derivation as highlighted on the center of Figure 12. The maximum peak acceleration was used as a reference to normalize the subsequent peak amplitudes and used as the time zero reference as shown on the right of Figure 12. An exponential trend line was included along with a correlation reference, $\mathrm{R}^{2}$, to observe how well the profile correlated test data. Typical correlation values should be above $95 \%$. The exponential trend line helps derive $\alpha$, which is used to calculate the logarithmic decrement, $\delta_{\text {log }}$, of an underdamped system. For this data set, $\alpha$ is -139.6 with a correlation of $96.17 \%$. The exponential functions helps quantify the rate of decay in the system and the general expression is describe in Equation 18.

$$
y=e^{-\alpha t}(18)
$$

The logarithmic decrement is used to find the damping ratio, $\xi$, in the time domain and is based on successive peak amplitudes, where $n$ is the peak amplitude and $n+m$ are the successive positive peak amplitudes away from $n$. If $m=1$, then $\delta_{\log }$ can be reduced as the following ${ }^{7}$ :

American Institute of Aeronautics and Astronautics 


$$
\delta_{\log }=\frac{1}{m} \ln \frac{x_{n+m}}{x_{n}}=\ln \frac{x_{2}}{x_{1}}=\frac{1}{\alpha}
$$

The damping ratio, $\xi$, and logarithmic decrement, $\delta_{\log }$, are related by the expression ${ }^{7}$,

$$
\xi=\frac{\delta_{\log }}{2 \pi}
$$

With all variables defined, the damping coefficient can be derived using equation 21 .

$$
c=2 * \xi * \sqrt{k * m}
$$

The same process is repeated for the 8-ft Nylon slings. A summary of the derived damping coefficients for CPAS

\begin{tabular}{|c|c|c|c|c|c|c|c|c|}
\hline $\begin{array}{c}\text { Data } \\
\text { Source }\end{array}$ & $\begin{array}{c}\text { Material } \\
\text { (Type) }\end{array}$ & $\begin{array}{c}\text { Alpha } \\
\text { (no units) }\end{array}$ & $\begin{array}{c}\text { natural frequency } \\
\text { (hz) }\end{array}$ & $\begin{array}{l}\text { Period } \\
\text { (s) }\end{array}$ & $\begin{array}{l}\text { Logarithmic Decrement } \\
\text { (no units) }\end{array}$ & $\begin{array}{l}\text { Damping Ratio } \\
\text { (no units) }\end{array}$ & $\begin{array}{c}\text { Critical Damping Coefficient } \\
\text { (lbf* } s \text { /inches) }\end{array}$ & $\begin{array}{c}\text { Damping Coefficient } \\
\text { (lbf*s/inches) }\end{array}$ \\
\hline Accel. (2000g) & Kevlar & 139.6 & 152.7925 & 0.0065 & 0.007163 & 0.001140 & 213.67 & 0.2436 \\
\hline SEF PIN (STBD) & Nylon & 45.5 & 29.6797 & 0.0337 & 0.021978 & 0.003498 & 41.51 & 0.1452 \\
\hline SEF PIN (PORT) & Nylon & 38.16 & 29.6337 & 0.0337 & 0.026205 & 0.004171 & 41.44 & 0.1728 \\
\hline
\end{tabular}
specific lines is shown in Table 4.

\section{Table 4: Damping coefficients}

\section{Linear Viscous and Rotational Damping}

Controlling the translational and rotational motion of hardware components in the simulation is required to remove numerical instabilities or artifacts that may arise during a nominal or Monte Carlo run. Flight objects like the 6:2 confluence and EPJD require rotational damping to prevent excessive over-rotation that produce unrealistic twisting in the lines. In Adams a three-component torque vector is used to apply a rotational force at the center of gravity to dampen rotational motion. The general torque vector expression is shown in equation 22, where $c_{r}$ is the rotational damping term used to stabilize the object during the transient event.

$$
\tau=-c_{r} * \omega(22)
$$

Single component forces are used to apply viscous damping coefficients associated with the objects velocity to dampen out oscillations. A snapshot of the torque vector (red) and linear viscous damping (blue) forces are shown in Figure 13.
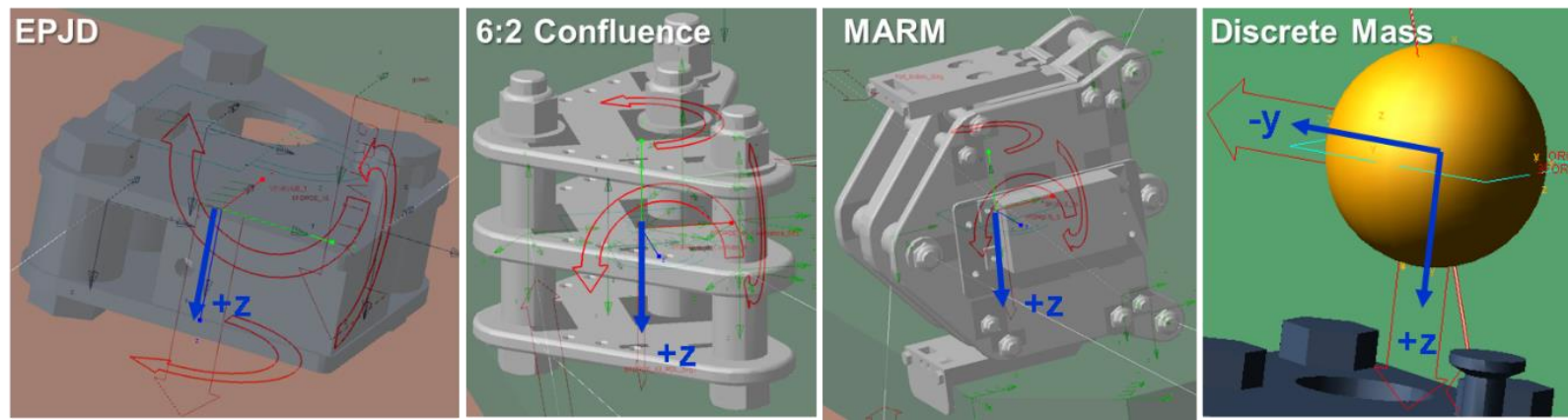

Figure 13: Rotational and Linear Viscous Damping

\section{Discrete Masses}

Discrete point masses were introduced to simulate the resistive inertial forces of the individual lines with the intent to decelerate the deployment sequence and match the timing as seen in-flight. With the addition of discrete point masses, attention was allocated to distributing mass along 10 point masses for the extraction line and two for the reposition deployment line and each harness sling. Ten point masses were settled on by scoping model development plus implementation time and required fidelity. This is sufficient to characterize the lines responsive motion when a drag force is applied to the system. The non-linear force-deflection curve and discrete point masses are used in the simulation concurrently and

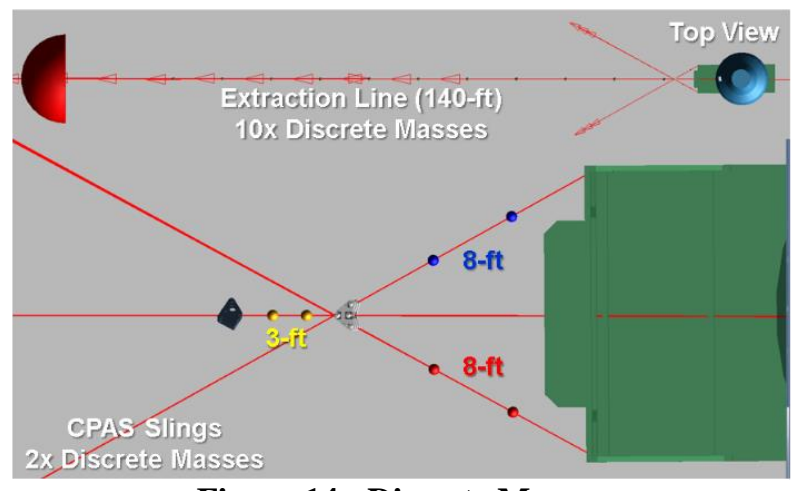

Figure 14: Discrete Masses 
deployed sequentially using a variety of strategically defined constraints and sensors. The results are representative loads predictions for lines used in the extraction system. The fully deployed discrete masses used in the simulation are depicted on Figure 14

\section{Hi-Fi Loads Predictions using Non-Linear Force Function, $\mathbf{P}(\mathbf{n}, \boldsymbol{\delta})$ and Damping, $\mathrm{c}$}

Preflight predictions leading into the EDU-A-CDT-3-15 drop test applied the novel non-linear force function, $\mathrm{P}(\mathrm{n}, \delta)$, with calculated damping coefficients, c, using Equation 21 and assumed a $\xi=1$. Figure 15 compares Adams Hi-Fi loads predictions with acquired test data measurements. High speed video snapshots are shown to help visualize the events occurring during this transient event. The Adams simulation over predicts the initial dynamic load experienced when the 3-ft Kevlar line becomes taut and pulls the 6:2 confluence at rest on the CPSS shelf (Event 13). The predicted time from Event 3-5 is faster compared to flight test data. This may be attributed to a combination of the simulation sling length being shorter than reality or the relative velocity of the confluence departure was faster compared to flight, thus the lines became taut faster. At Event 6, the extraction system lines are static and the simulation predictions were in line with the Single Event Fitting load pins located at the attach locations on the backstop.
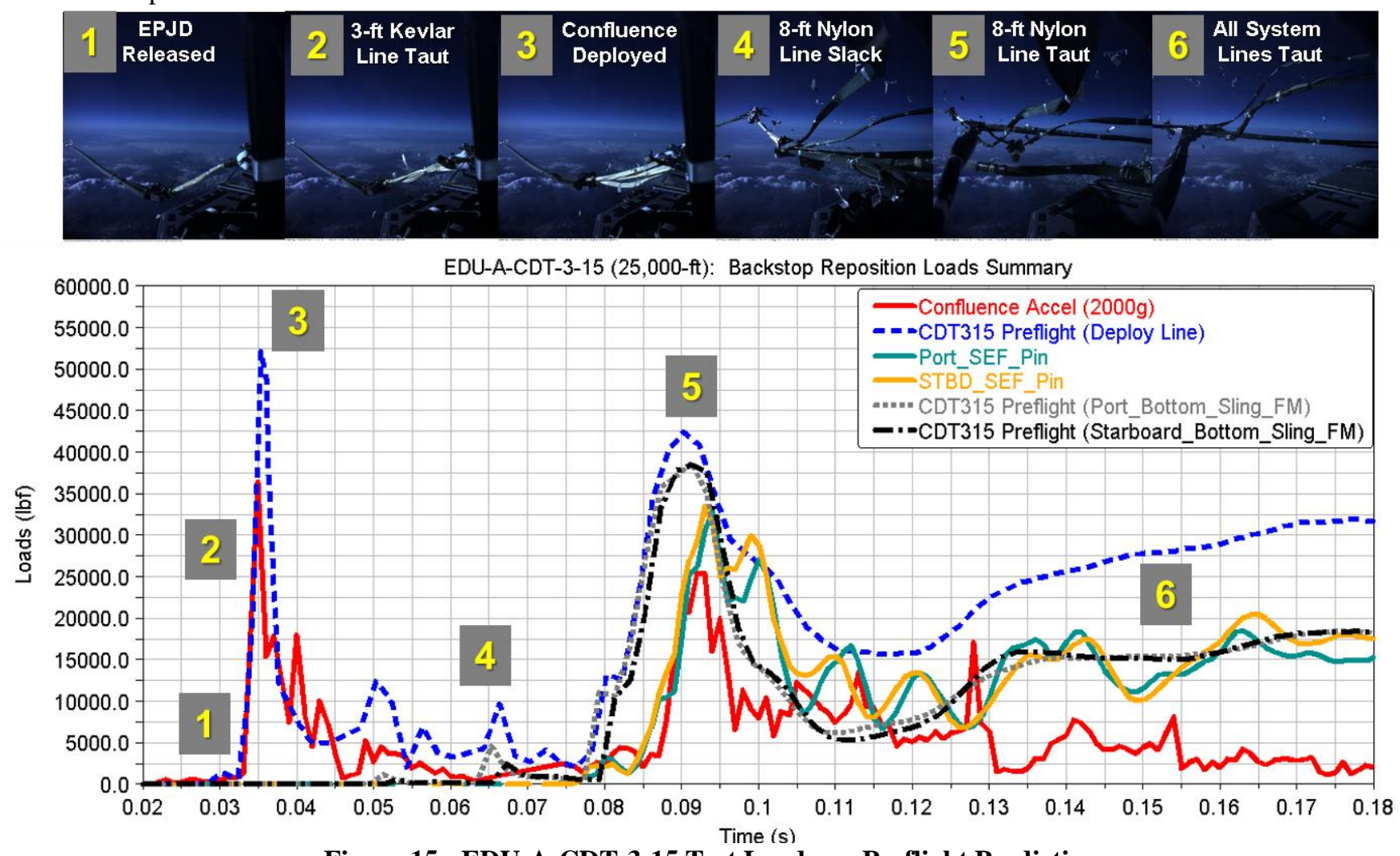

Figure 15: EDU-A-CDT-3-15 Test Loads vs. Preflight Predictions

\section{Extraction Parachute Modeling}

\section{A. Extraction Parachute Center of Pressure and Center of Mass}

The analysis performed for the first PTV pre-flight prediction had the extraction parachute center of gravity $\left(\mathrm{CG}_{\text {ext }}\right)$ and pressure $\left(\mathrm{C}_{\mathrm{p}}\right)$ located on the parachute centerline at the canopy skirt. A sensitivity study conducted as part of the EDU-A-CDT-3-5 post-test assessment resulted in a decision to move the $\mathrm{C}_{\mathrm{p}}$ four inches aft of the canopy skirt to reduce instabilities in the simulated extraction parachute behavior observed during Monte Carlo runs. Starting with EDU-A-CDT-3-7, the extraction parachute center of pressure, $C_{p}$, was derived using mass properties and concepts described in the Parachute Dynamics and Stability Analysis ${ }^{1}$ report. The report defines the $\mathrm{C}_{\mathrm{p}}$ for the SRB Drogue Baseline Configuration as a function of the parachute reference diameter, $D_{0}$. The canopy is assumed to be a thin hemispheric shell and the projected diameter, $D_{p}$, for a conical textile parachute is proportional to $D_{o}$ as shown in Equation $23^{2}$

American Institute of Aeronautics and Astronautics 


$$
D_{p}=0.70 * D_{o}(23)
$$

If the parachute is reefed with a reefing line length, $\mathrm{L}_{\mathrm{r}}$, then the reefed diameter is substituted for the reference diameter. The reefed diameter, $D_{R}$, is determined from the reefing-line length according to Equation 24 .

$$
D_{R}=\frac{L_{r}}{\pi}(24)
$$

Figure 16 illustrates the resulting $\mathrm{C}_{\mathrm{p}}$ and $\mathrm{CG}_{\mathrm{ext}}$ locations. The length a, is one-half of the projected diameter, $\mathrm{c}$ is the

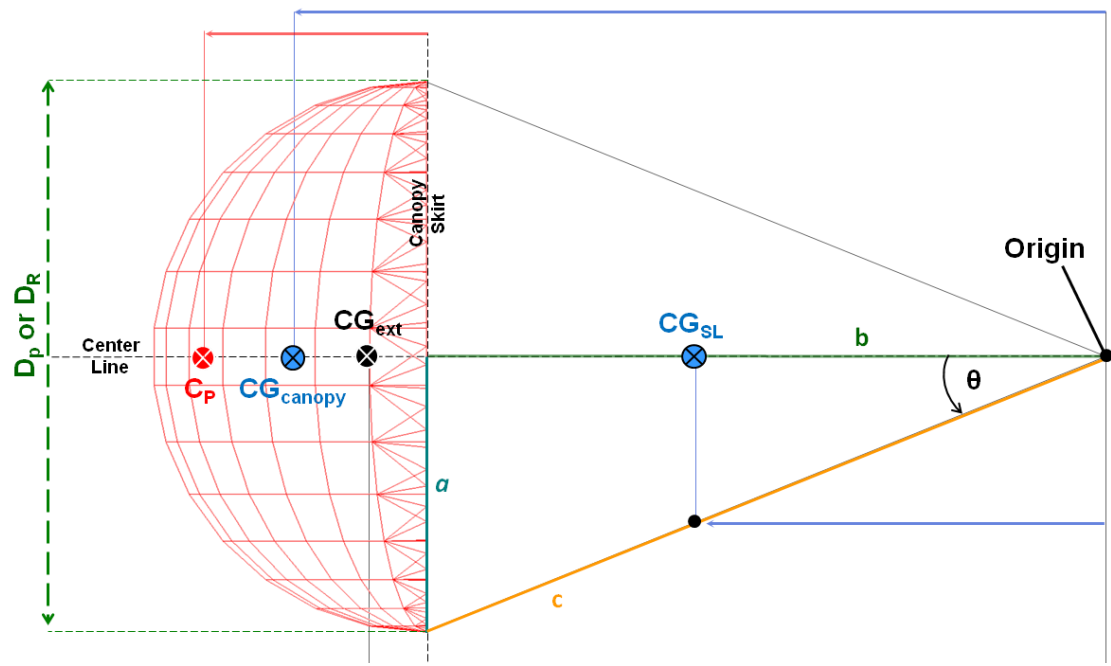

Figure 16: Extraction Parachute Centers of Pressure and Gravity Locations length of the suspension lines and $\mathrm{b}$ is calculated using the Pythagorean Theorem.

The $\mathrm{C}_{\mathrm{p}}$ is placed a distance aft of the canopy skirt along the parachute centerline and is equal to $16.3 \%$ of $D_{p}$ as shown in Equation 25.

$$
C_{p}=0.163 * D_{p}
$$

The center of gravity of the canopy, $\mathrm{CG}_{\text {canopy, }}$ and suspension lines, $\mathrm{CG}_{\mathrm{SL}}$, are used with their respective masses, $\mathrm{M}_{\text {canopy }}$ and, $\mathrm{M}_{\mathrm{SL}}$, to determine the $\mathrm{CG}_{\text {ext }}$ as shown

in Equation 26.

$$
C G_{\text {ext }}=\frac{C G_{S L} M_{S L}+C G_{\text {Canop }} M_{\text {Canopy }}}{M_{\text {total }}}, \text { where } C G_{S L}=\frac{c * \cos (\theta)}{2} \text { and } C G_{\text {Canopy }}=b+\frac{D_{p}}{4}(26)
$$

\section{B. Extraction Parachute Inflation Model}

The original inflation model, used for the first two PTV tests used a table look up of drag area versus time based on heritage UD-233A data. Additionally, the drag area reduction factor, $\mathrm{K}_{\mathrm{ext}}$, originally introduce to account for the observed reduction of cluster drag area is no longer used. The latest Adams simulation uses a parachute inflation model, with the average drag area inferred from on-board accelerometer data ${ }^{3}$.

1. Drag Area Growth Curve for an Infinite and Finite Mass

For a given parachute stage $i$, the model defines the inflation start time, $t_{i}$, with respect to bag strip and is defined as the point when the apex of the canopy exits the deployment bag. The drag area increases from an initial value, $\left(\mathrm{C}_{\mathrm{D}} \mathrm{S}\right)_{i-1}$, at $t_{i}$, reaches a local maximum at $\left(\mathrm{C}_{\mathrm{D}} \mathrm{S}\right)_{\text {peak }}$, and then decreases to its steady-state value of $\left(\mathrm{C}_{\mathrm{D}} \mathrm{S}\right)_{i}{ }^{2}$. A generalized shape is illustrated in Figure 17. It should be noted that parachute peak load increases with increasing drag area slope.

Since the modeled extraction parachutes do not disreef, the drag area growth curve for finite mass parachutes are reduced and shown in equation 27.

$$
\left(C_{D} S\right)(t)=\left(C_{D} S\right)\left(\frac{\left(t-t_{i}\right)}{t_{f}}\right)^{\text {expopen }}
$$

The fill time, $t_{f}$, is a function of the fill constant, $n$, nominal parachute diameter, $\mathrm{D}_{\mathrm{o}}$, and initial velocity, $\mathrm{V}_{\mathrm{i}}$.

$$
t_{f}=\frac{n * D_{0}}{V_{i}}(28)
$$

The equations modeling an infinite mass inflation take into account and over inflation factor. The over inflation factor is the ratio of the peak drag area to the steady state value shown in equation (29). For finite mass inflation $C_{k}$ is

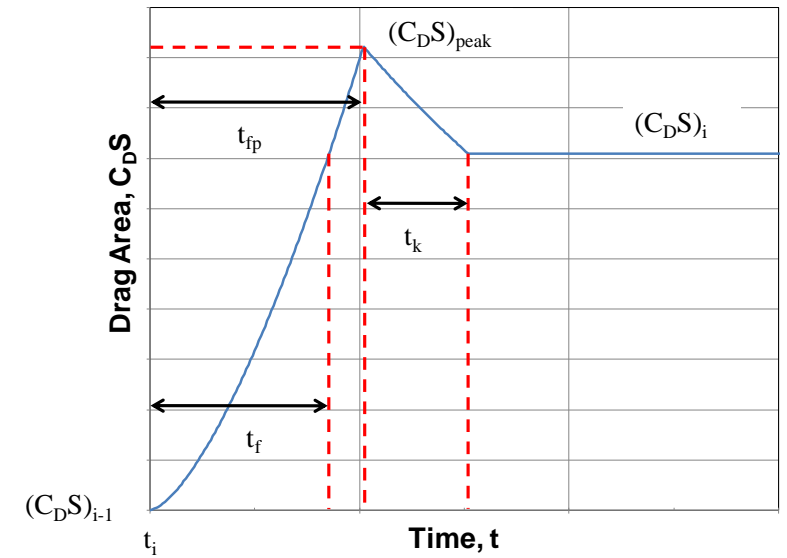

Figure 17: Drag area growth curve assumed equal to 1 .

American Institute of Aeronautics and Astronautics 


$$
C_{k}=\frac{\left(C_{D} S\right)_{\text {peak }}}{\left(C_{D} S\right)_{i}}(29)
$$

The amount of time it takes to ramp down from the peak drag area to steady-state drag area is defined as $\mathrm{t}_{\mathrm{k}}$. The drag area during the ramp down period is defined in Equation 30.

The time to peak drag is, $t_{f p}$ is defined as

$$
\left(C_{D} S\right)(t)=\left(C_{D} S\right)_{\text {peak }} *\left(C_{K}\right)^{\left(t_{f p}+t_{i}-t\right)} / t_{k}
$$

$$
t_{f p}=t_{f} *\left(\frac{\left(C_{k^{*}}\left(C_{D} S\right)_{i}\right)-\left(C_{D} S\right)_{i-1}}{\left(C_{D} S\right)_{i}-\left(C_{D} S\right)_{i-1}}\right)^{1 / \text { expopen }}
$$

Integrating the parachute inflation model combined with observed inflation start times from test video on-board the aircraft, an improved estimation of the extraction line load is achievable. Figure 18 illustrates the extraction parachute modeling upgrades by compares preflight (gray), test data (blue) and post-flight reconstruction (red) trajectories of the extraction chute drag area growth curve and extraction line load, respectively. The extraction line load is the product of the mated vehicle longitudinal acceleration and weight. The drag area growth curve represents the line load over the instantaneous dynamic pressure (derived from measured atmospheric data). The inflation parameters: fill constant $(n)$, opening exponent (expopen) and over-inflation factor $\left(C_{k}\right)$ are determined using a MatLab optimization script which correlates to the test data inflation profile.
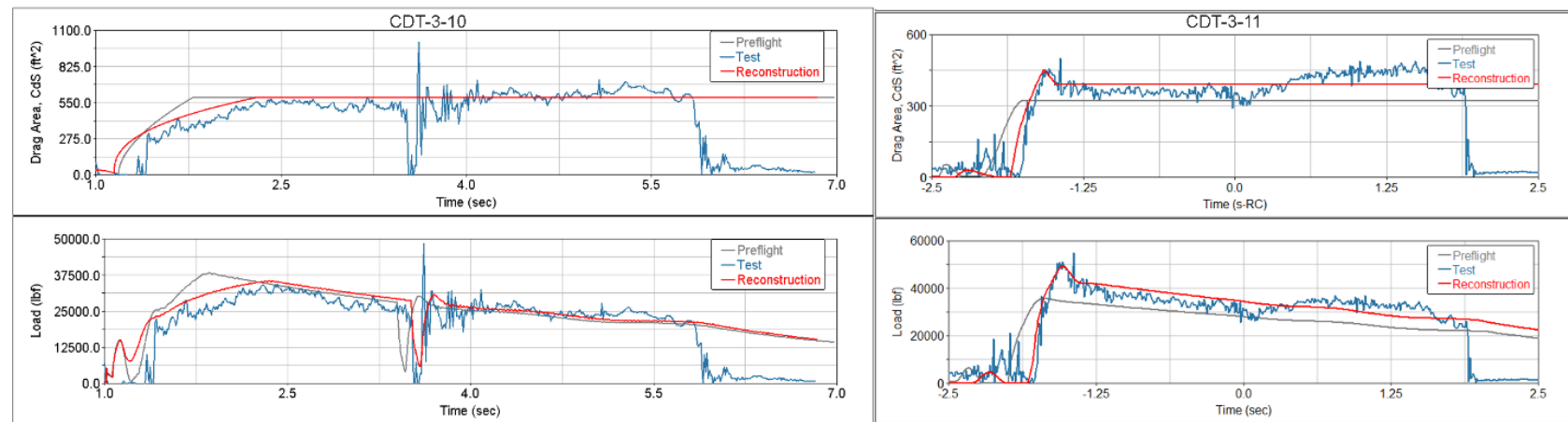

Figure 18: Extraction Parachute CdS and Loads Comparison for CDT-3-10 and CDT-3-11

\section{Correlating Extraction Parachute Performance While on the Aircraft Ramp}

The second step in the standard reconstruction process is correlating the extraction sequence from green light through the end of ramp. While onboard the aircraft the mated vehicle is locked in until the pull force in the extraction line exceeds $1 / 2 \mathrm{~g}$. Until this constraint is met, the mated vehicle remains at rest and the deployed extraction parachutes progress on with the inflation process. The free body diagram for this phase of flight is shown in Figure 19 and accounts for the friction force applied by the ramp.

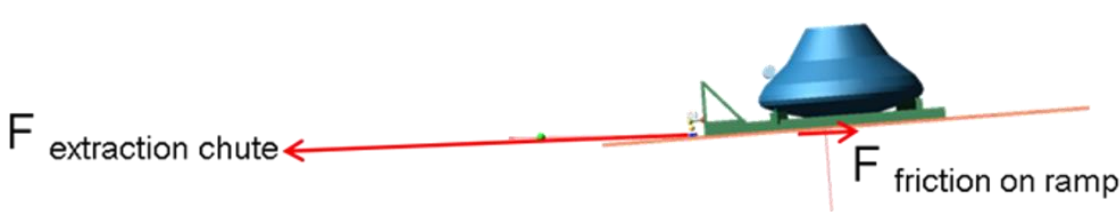

\begin{tabular}{|r|c|}
\hline Sequence of Events on Ramp & Time (s-RC) \\
\hline Green Light & $-3.5 \mathrm{sec}$ \\
\hline Extraction Parachute Bag Strip & $-2.5 \mathrm{sec}$ \\
\hline First motion & $-2.2 \mathrm{sec}$ \\
\hline Aft Edge of CPSS off Ramp & $-0.4 \mathrm{sec}$ \\
\hline Ramp Clear & $0.0 \mathrm{sec}$ \\
\hline
\end{tabular}

Figure 19: Extraction Free-Body Diagram

The translational equation of motion during this phase is described by Equation 32, where the governing force while on the ramp is the extraction parachute drag force.

$$
F_{\text {extraction chute }}-F_{\text {friction on ramp }}=m_{\text {mated }} \ddot{X}_{\text {mated }}, \text { where } F_{\text {extraction chute }}=\bar{q} C_{D} S(32)
$$

As a result, when reconstructing performance while on the ramp, the extraction parachutes are activated by $\mathrm{t}_{\mathrm{i}}$, at a time earlier than the acceleration of the mated vehicle and a larger drag force is applied to better match the $C_{d} S$ post ramp clear. This technique can be seen in Figure 18 for EDU-A-CDT-3-10.

\section{Modeling Vehicle Extraction and Smart Separation Performance}

\section{A. Defining a Smart Separation Window}

Mathematically bounding vehicle dynamics with a logic function has proved to be repeatable. The key parameters used to sense favorable dynamics is time, pitch attitude and pitch rate. In flight, the optimal path entering the window is at the center of the maximum pitch rate window. The conceptual figure below shows the different scenarios that

American Institute of Aeronautics and Astronautics 
help define a smart separation window. Selecting a smart separation window must be assessed in a time, pitch, pitch rate and angle of attack domain. The red box is the pitch-pitch rate domain that is made up of inputs used in the avionics system. This domain is optimized by shifting the window horizontally and vertically to bound favorable vehicle dynamics. The blue box is the angle of attack domain of the PTV after separation and is impacted by the pitch-pitch rate domain. The three PTV hand-over scenarios are: apex forward $\left(\alpha<90^{\circ}\right)$, heat shield forward $\left(90^{\circ}<\alpha<150^{\circ}\right)$, and over-rotating heat shield forward $\left(\alpha>150^{\circ}\right)$. The PTV hand-over state is then delivered to various simulations to complete preflight analyses shown in the green box.

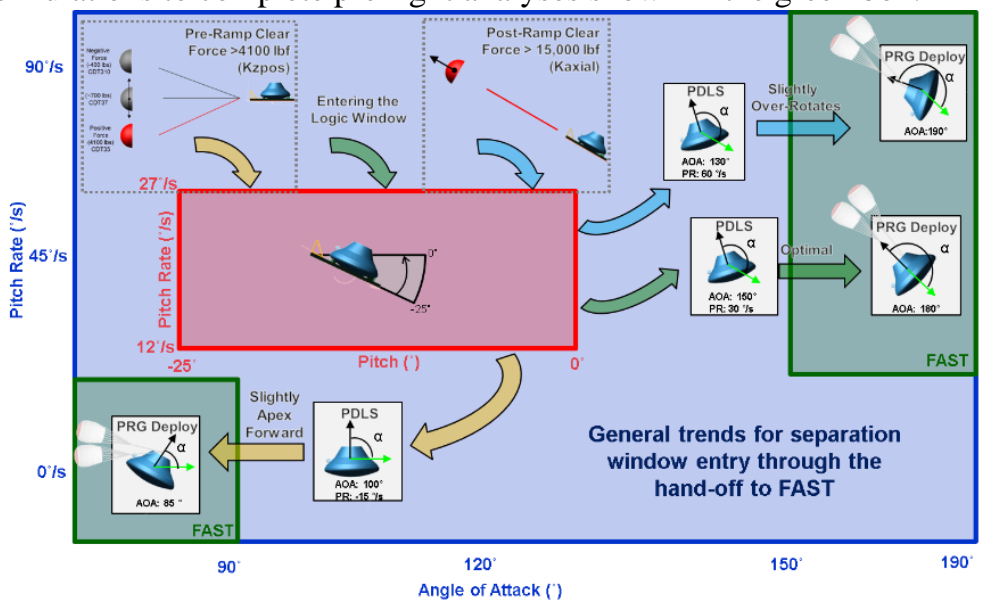

Figure 20: Defining a Smart Separation Window

\section{B. Standard Load Transfer Overview}

A standard extraction parachute-toCPSS load transfer test technique was successfully used for five PTV flight tests. The technique applies an extraction parachute drag force at the EFTC attach assembly on the CPSS from extraction parachute deployment to a defined time after the PTV has released (typically $135 \mathrm{~s}-$ $\mathrm{RC})$. During the first $0.5 \mathrm{~s}$ from ramp clear, the mated vehicle experiences a tip-off angle and rate and performs its initial gravity turn while attached to two $28-\mathrm{ft}$ extraction parachutes. The mated vehicle proceeds to pitch to a minimum downward pitch angle that is on average $60^{\circ}$ and $70^{\circ}$

for 25,000-ft and 35,00-ft extraction altitudes, respectively. At the completion of the gravity turn, the mated vehicle takes about $1.0 \mathrm{~s}$ to reach its maximum pitch rate which ranges from $30 \% \mathrm{~s}$ to $60 \%$. After this maximum pitch rate is reached, the pitch plane dynamics (angle and rate) nominally enter a pre-programmed window of conditions that are conducive to a successful separation. When the avionics system detects that the conditions have been achieved, it sends a signal to the CPSS to activate the three pyrotechnique steel blades that cut vectran cords used to attach the PTV to the CPSS at ball-and-socket interfaces. There is a system lag between the time the command is issued and the physical separation of the bodies due to an avionics processing delay and the time required to overcome friction at the ball-socket attach locations. Upon release, friction at the ball and socket interfaces delays separation slightly (average total lag in the system is $60 \mathrm{~ms}$ ). Following the physical separation, the CPSS is pulled away from the PTV by the extraction parachutes, and at a separtion distance of approximately $31-\mathrm{ft}$, the programmer parachutes are deployed. Elapsed time from physical separation to programmer inflation is approximately 1.2 seconds. During this time the PTV rotates heat shield forward. Control authority is regained at the instant the programmers are fully inflated.

\section{Backstop-Reposition Overview}

At the completion of EDU-A-CDT-3-11, post flight inspection of the hardware revealed damage to the EPJD and aft CPSS attachment. This was attributed to a bending moment which occurred between extraction and separation. The EFTC is free to swing upward, but a hard stop prevents it from rotating below the CPSS platform, and the extraction parachutes will align with the local velocity vector. Therefore, a moment is created when the extraction line creates a negative angle of attack relative to the CPSS during the pitchdown motion in the first second after ramp clear. This is illustrated in Figure 21. The bending moment is proportional to the extraction parachute force and the angle of attack. The extraction force during CDT-3-11 (extracted at 35,000 ft) was $39 \%$ higher than previous tests (from $25,000 \mathrm{ft}$ ) at the time of peak load (just

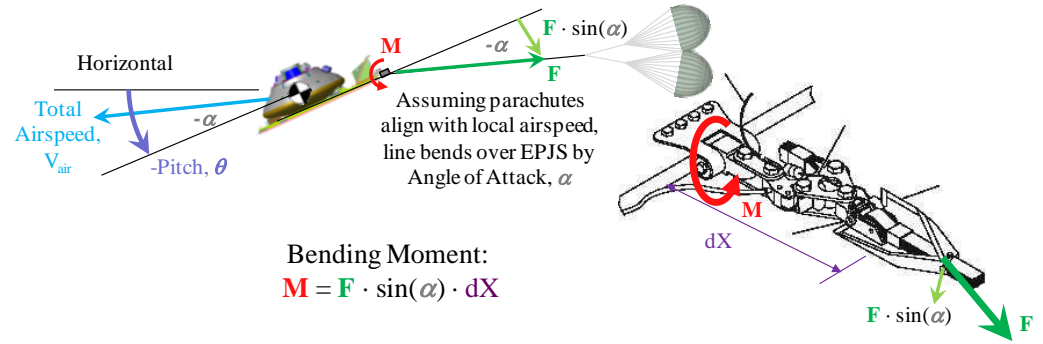

Figure 21: Bending Moment on Extraction System after first motion), and the force was $28 \%$ higher than previous tests during the maximum pitch-down event. 
As a result of the EDU-A-CDT-3-11 bending moment anomaly, a new event was introduced that would distribute the extraction load line of action to the four corners of the CPSS backstop by performing the reposition technique immediately following ramp clear, as opposed to doing it during the CPSS descent. At ramp clear, the EPJD is released in the load path of the extaction line and the 3-ft reposition deployment line subsequently becomes taut and transmits a transient force to the confluence mounted on the CPSS shelf. The confluence reacts to the dynamic load as it departes at $140 \mathrm{ft} / \mathrm{s}$ (varies depending on extraction altitude). A second transient load occurs when the 8-ft slings attached to the confluence become taut, at which time the system is at its full stretch length and the motion dampens out shortly after this state has been reached. A summary of the Adams modeling of this event is shown in Figure 22.

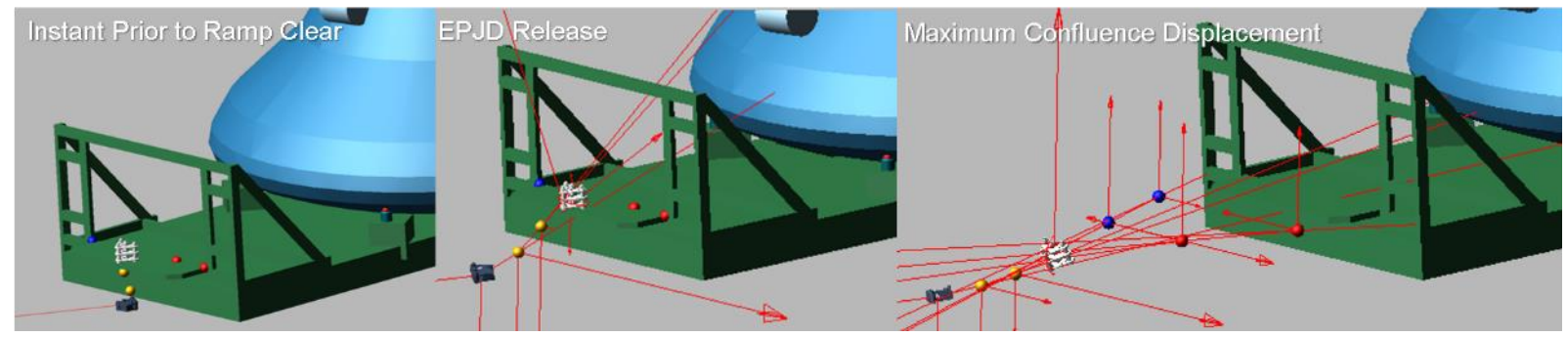

Figure 22: Simulating Backstop Reposition Sequence

\section{Reconstruction Process Using External Extraction Parachute Forces to Correlate Dynamics}

Producing flight correlations in the time domain was the primary objective in the first edition of this paper ${ }^{2}$.The objective included producing a time window which opened at $1.0 \mathrm{~s}-\mathrm{RC}$ to ensure safe proximity distances from the aircraft before any mid-air manuever was allowed and a window close at $4.0 \mathrm{~s}-\mathrm{RC}$ to release in the event of an offnomial extraction.

1. Extraction Parachute Axial and Downdraft Force (External Forces)

During the EDU-A-CDT-3-10 post-test reconstruction, a modeling deficiency was identified when the Best Estimate Trajectory (BET) was compared with Monte Carlo predictions (green points) entering the smart separation logic window. The critical observation was that the preflight predictions did not capture the true flight dynamics (red trace), Figure 23. The simulation was not capable of bounding the true flight dynamics without the introduction of a time varying axial force applied on the extraction parachute center of pressure, $\mathrm{C}_{\mathrm{p}}$. The axial force was specific to the test

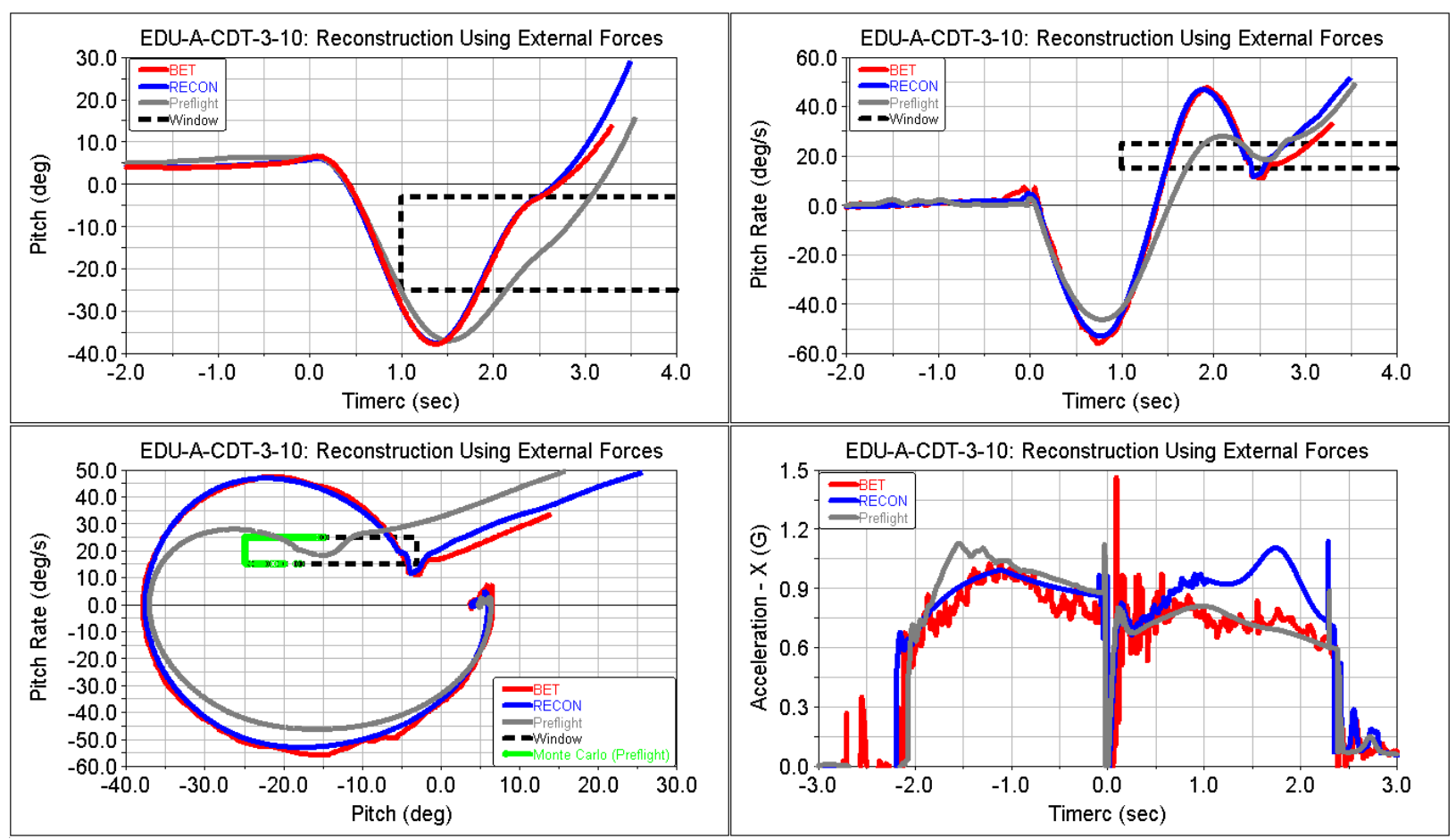

Figure 23: Reconstructing using External Forces

American Institute of Aeronautics and Astronautics 
and provided exceptional correlations through the separation event, $2.2 \mathrm{~s}-\mathrm{RC}$. After the separation event, $2.5 \mathrm{~s}-\mathrm{RC}$, the model would deviate from the BET and over-predict the hand-over conditions at Programmer deployment line stretch.

Preflight analysis leading into EDU-A-CDT-3-14, now included the introduction of two external forces: downdraft and axial forces acting on the extraction parachute $C_{p}$. Each served a specific purpose. The downdraft
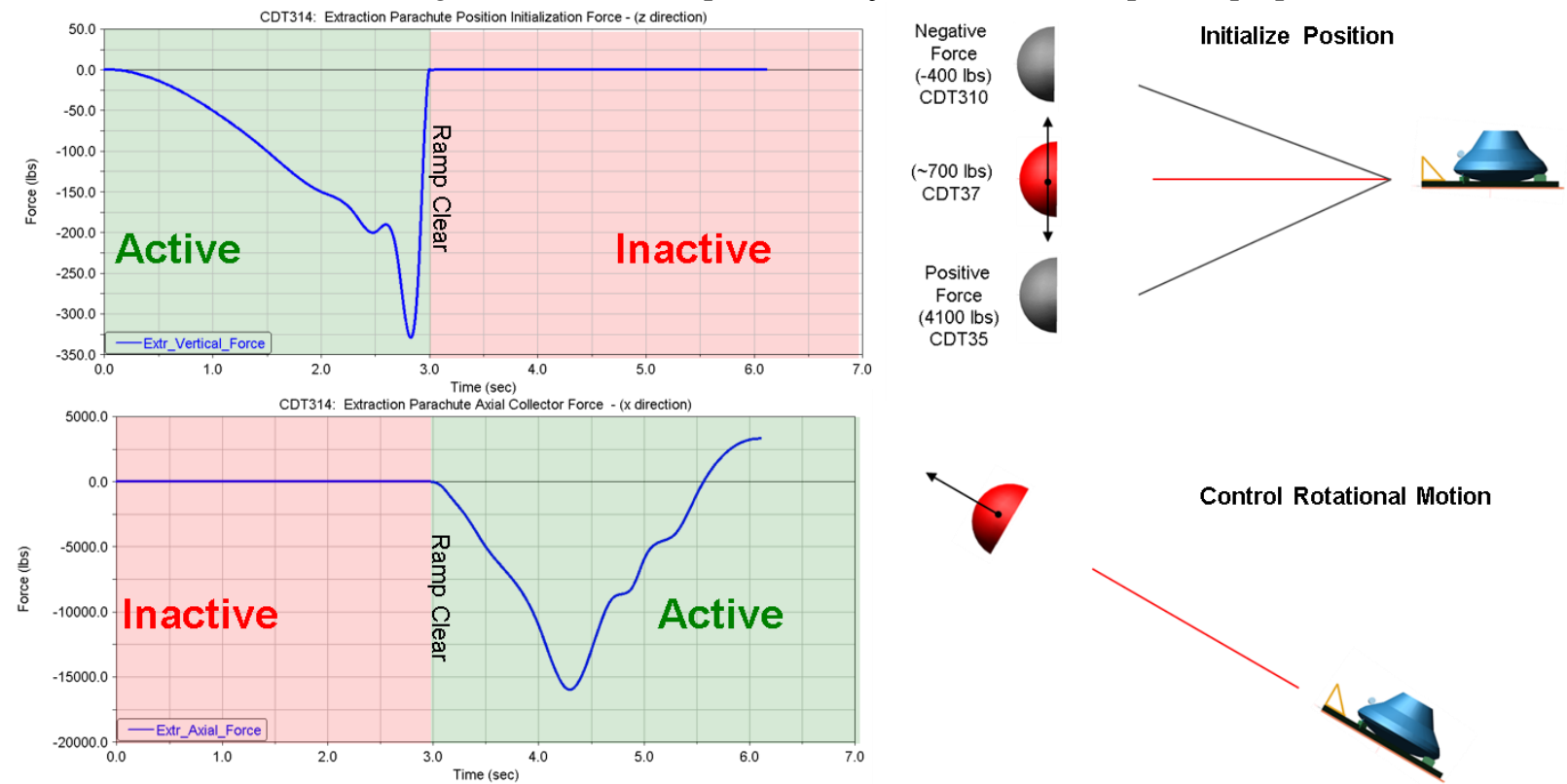

Control Rotational Motion

Figure 24: Applied External Forces

force, applied inside the aircraft, allowed the simulatin to initialize the position of the extraction parachutes below, inline, or above the aircraft ramp angle. The axial force, applied outside the aircraft, allowed the simulation to control the rotational motion and match the true flight dynamics entering the right side of the smart separation logic window. Each external force is time varying, requires retuning to specific extraction altitudes and are defined as a pre and post ramp clear force. The nominal force profiles are shown in Figure 24 with application locations at the $\mathrm{C}_{\mathrm{p}}$. The pre ramp clear force range is [-400 lbf to $4500 \mathrm{lbf}]$ with $4500 \mathrm{lbf}$ being derived by EDU-A-CDT-3-5. The post ramp clear force profile was derived using EDU-A-CDT-3-10 and is dispersed 50\%, which results in Monte Carlo cases over-shooting the right side of the window. The 50\% disperison was settled on to account for the unknowns at the time. The expense for matching flight dynamics using external forces was a reduction of fidelity in the loads predictions. The signature of the post ramp clear force can be seen in the acceleration plot between $1.5 \mathrm{~s}-\mathrm{RC}$ and 2.0 $\mathrm{s}$-RC. No aerodynamic forces were tuned.

Prior to EDU-A-CDT-3-15, a simplified model was employed using a massless 2-point link along with the external forces mentioned. Since the test failure cases, the model has evolved to match reality and the latest version used is the Hi-Fi model with an EPJD, 6:2 confluence and discrete masses. The subsequent sections use the 6:2 confluence model and aerodyanmic parameters to produced flight correlations. No external forces are used in the Hi-Fi reconstructions. Loads are derived using the non-linear force function, $\mathrm{P}(\delta, n)$.

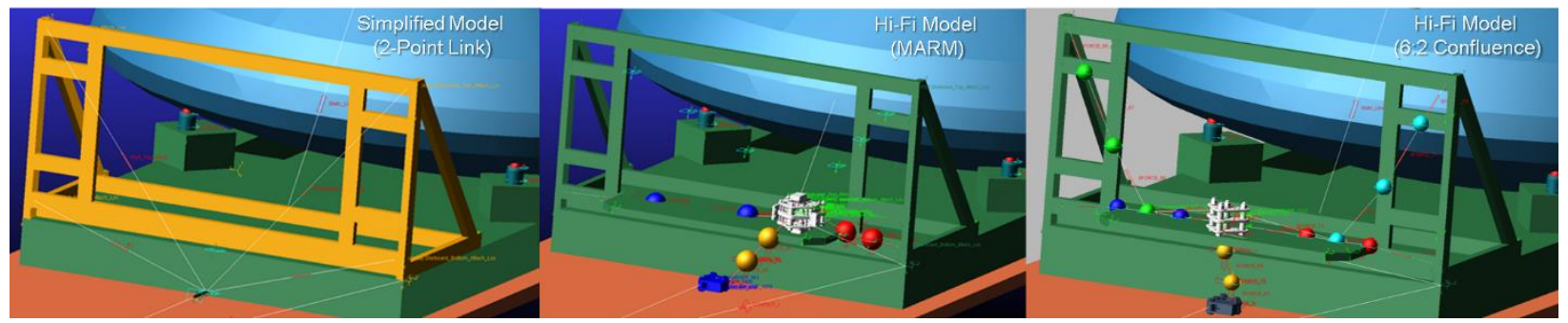

Figure 25: Evolution of Adams Modeling 


\section{E. Pitch Domain Performance}

The following reconstruction results focus on tuning aerodynamic parameters discussed in the aerodynamics section to match flight dynamics. No external forces are applied to maintain loads fidelity in the simulation. Figure 26 summarizes pitch attitude dynamics for all PTV tests to date. For each flight test, the ramp modeling has produced good correlations with initial ramp angles ranging from $4^{\circ}-6^{\circ}$ while on the aircraft $(2.0 \mathrm{~s}-3.5 \mathrm{~s})$. The pitch damping derivative while in the aircraft wake, Mated_cmq_wake, help match the minium pitch attitude which ranges from $30^{\circ}$ to $-45^{\circ}(3.5 \mathrm{~s}-4.5 \mathrm{~s})$. Outside the aircraft wake, Mated_cmq, helps match the next phase of flight $(4.5 \mathrm{~s}-5.5 \mathrm{~s})$ as the mated vehicle begins its upward pitch motion prior to entering the smart separation window $\left(-5^{\circ}\right.$ to $\left.-11^{\circ}\right)$. The final phase of flight $(5.5 \mathrm{~s}-6.5 \mathrm{~s})$ is the PTV separating from the CPSS through the programmer deployment line stretch event and hands-over to the programmer phase. The only 35,000 -ft test to date is EDU-A-CDT-3-11. The aircraft airspeed at 35,000-ft increases to $550 \mathrm{ft} / \mathrm{s}$ from $350 \mathrm{ft} / \mathrm{s}$. The pitch profile follows the same behavior but the event sequences occur about $1.0 \mathrm{~s}$ faster compared to $25,000-\mathrm{ft}$.
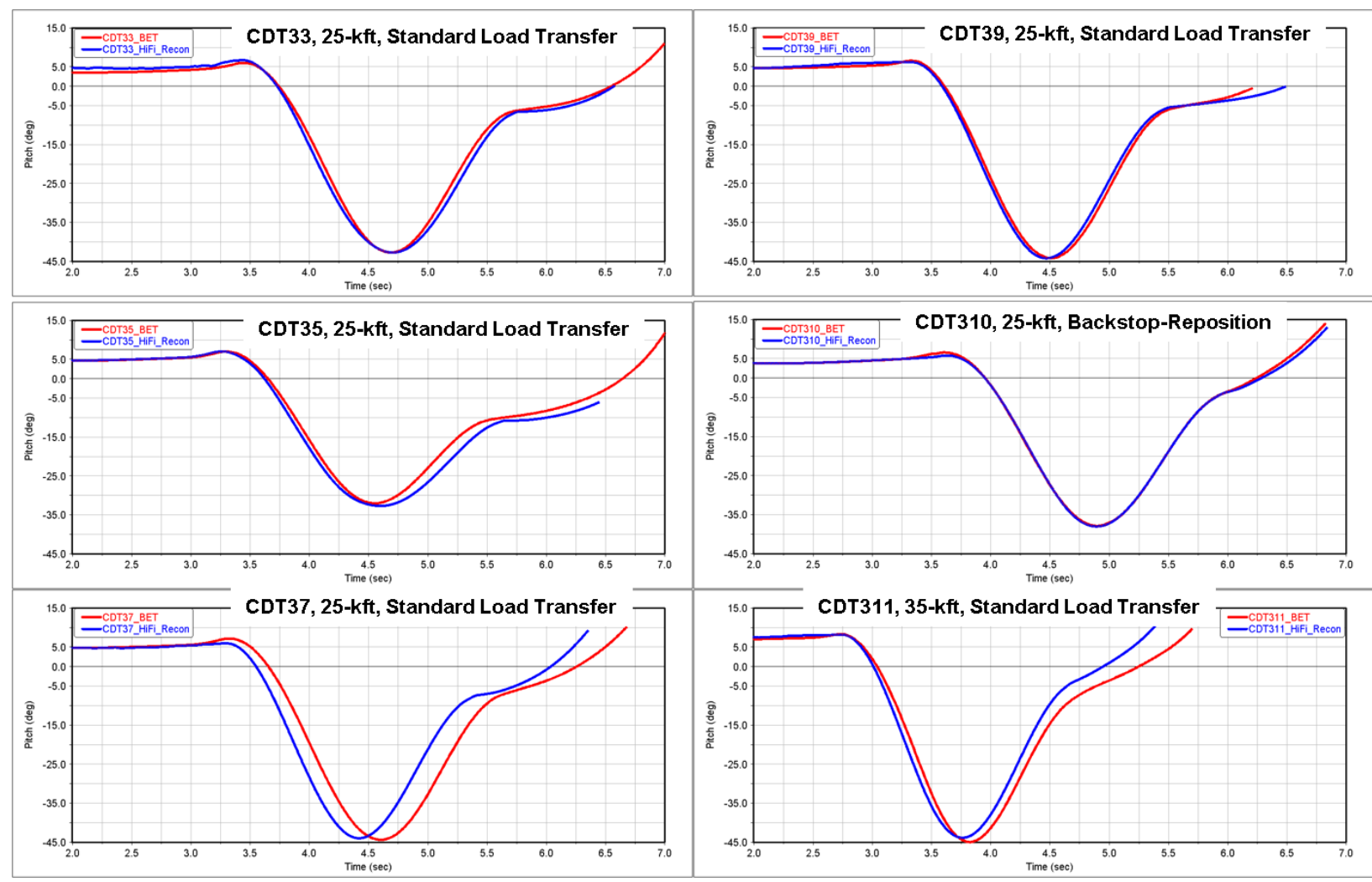

Figure 26: Pitch Angle Summary

\section{F. Pitch Rate Domain}

The simulation has also produced improved pitch rate flight correlations. The challenge during post-test reconstructions prior to the modeling upgrades included matching the initial tip-off pitch rate motion at ramp clear, the minimum and maximum pitch rate amplitudes and the hand-over condition to the programmer phase. Similar to the pitch profile, the aerodynamic variables were tuned to match the minimum and maximum pitch rates with exceptional results. Previously, the maximum pitch rate of the trajectory could not be matched and can be seen in the gray preflight trajectorty for EDU-A-CDT-3-10 (Figure 23), which would nomially reach a maximum of $30 \%$ (off $15 \%$ ). The introduction of the KCm_ptv multiplier used in the PTV alone pitch damping calculation has helped reduce the pitch rate at the end of the simulation to deliver flight like attitudes for PTV trajectory analysis $(6.25 \mathrm{~s}-$ $6.75 \mathrm{~s})$. The tip-off pitch rate motion ( $3.25 \mathrm{~s}-3.5 \mathrm{~s})$ at ramp clear is still a challenge to correlate and the authors speculate that there is a moment that is being experienced during this event that the simulation does not accout for at this time.

American Institute of Aeronautics and Astronautics 

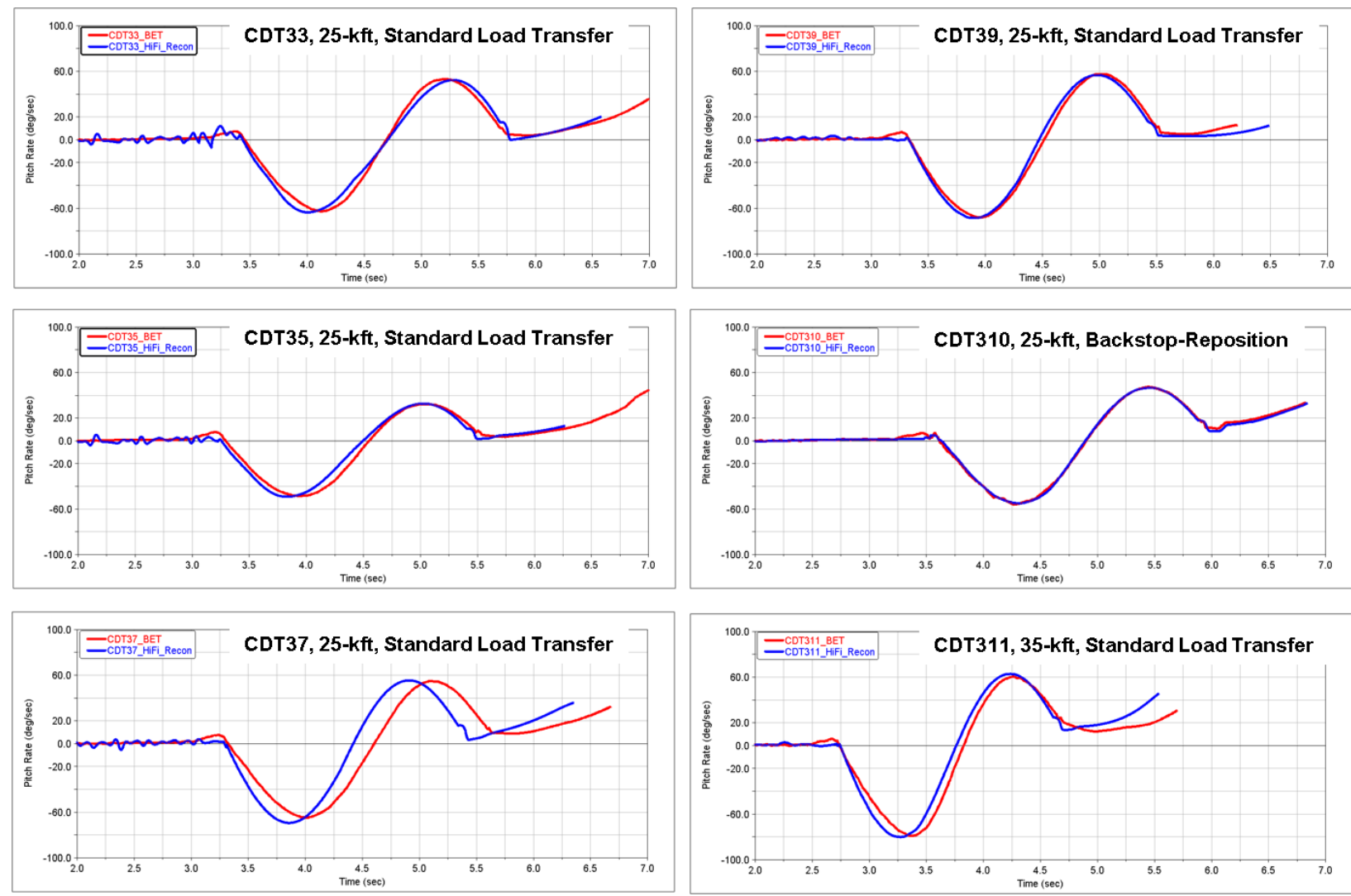

Figure 27: Pitch Rate Summary

\section{G. Pitch-Pitch Rate Domain}

The post-test reconstruction success criteria has evloved since the first edition. Prior to EDU-A-CDT-3-10, a successful reconstruction was primarily based on matching the separation time. The true measure of success is based on how well you match dynamics in the pitch-pitch rate domain. When the pitch-pitch rate domain is correlated first, the time domain dynamics align and usually are within $\pm 0.2 \mathrm{~s}$ at the separation event. Each PTV test to date has been revisited with the pitch-pitch rate correlation set as the primary objective. The attitude at which the mated vehicle enters the smart separation window is more critical than the time entry. It is more acceptable to be off-set in time versus attitude. The gray trace from the preflight prediction of EDU-A-CDT-3-10 (Figure 23), depicts a good match in time, but enters the left side of the pitch-pitch rate logic window compared to the flight trajectory. This test was a driver for evolving reconstruction objectives. As seen in the test summary below, exceptional results have been achieved in matching pitch-pitch rate dynamics. The tip off motion, between a pitch attitude of $3^{\circ}-7^{\circ}$, is still a target area for improvement, but the authors have collectively agreed that this is a lower priority and modeling solutions are continuing to be assessed to account for the pitch rate off-set. Contact modeling between the PTV/CPSS interfaces is also continually being investigated to produce more precise matches during the force interactions experienced at separation. Overall, the widest trajectory magnitude observed occurred at 35,000-ft for EDU-A-CDT-3-11. Conversely, EDU-A-CDT-3-5 observed a more compressed trajectry and is attributed to the downward position of the extraction parachutes at ramp clear.

American Institute of Aeronautics and Astronautics 

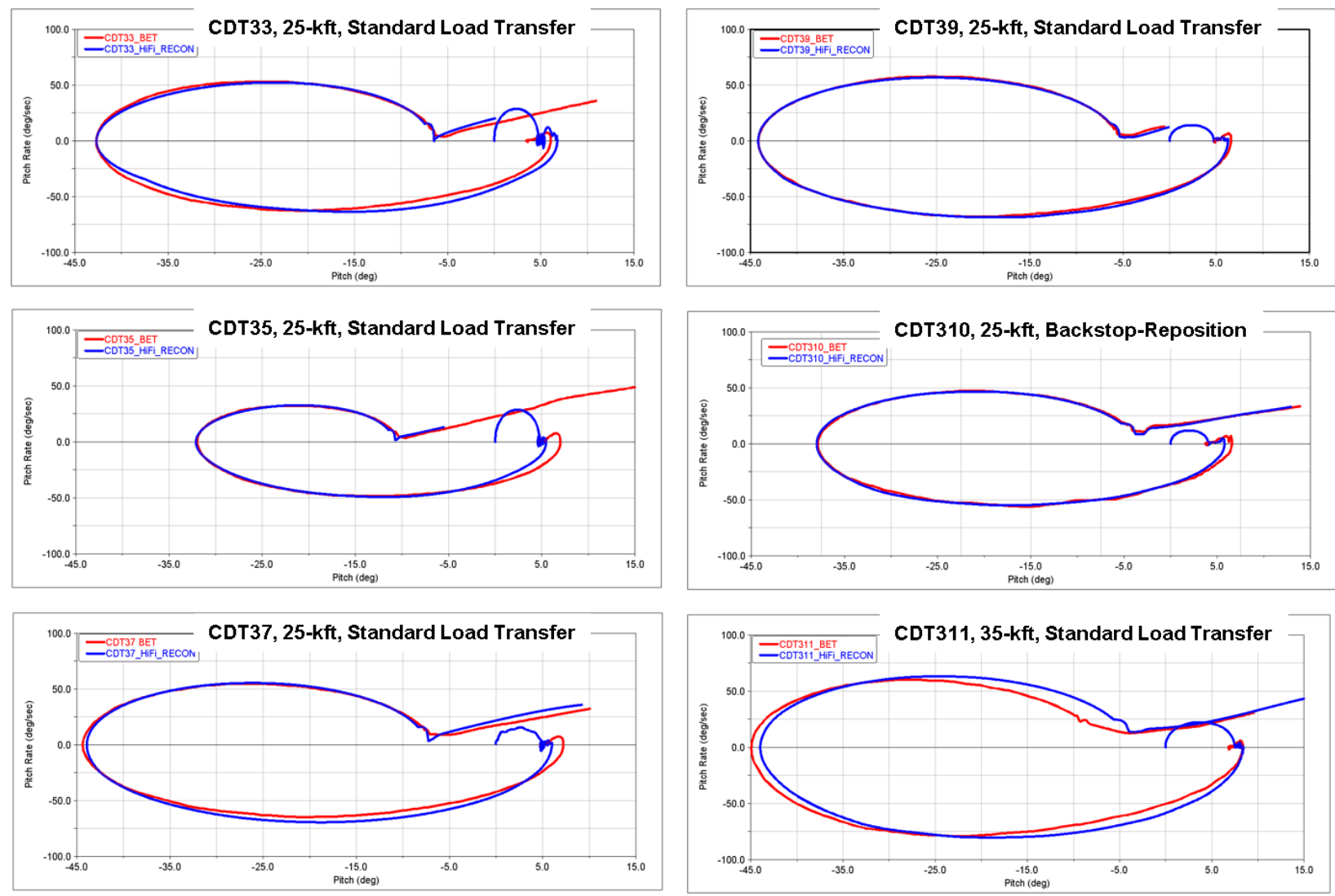

Figure 28: Pitch-Pitch Rate Summary

\section{H. EDU-A-CDT-3-15}

The most recent test in the CPAS EDU test campaign, EDU-A-CDT-3-15, was successfully performed from 25,000 - $\mathrm{ft}$. The backstop reposition technique was used and the pitch-pitch rate time history recorded by the avionics system is shown in red. Pre-test analysis involved a concurrent effort to execute two extraction hardware models in Adams. The first, referred to as the "Hi-Fi" model, was to produce a representative loads prediction using a finite element approach. The second, referred to as the "simple" model, involved the model used in recent previous tests which was known to produce good dynamics predictions but deficient loads predictions. Because the Hi-Fi model was still in development, the simple model was used to produce the official test predictions. The test was instrumented to

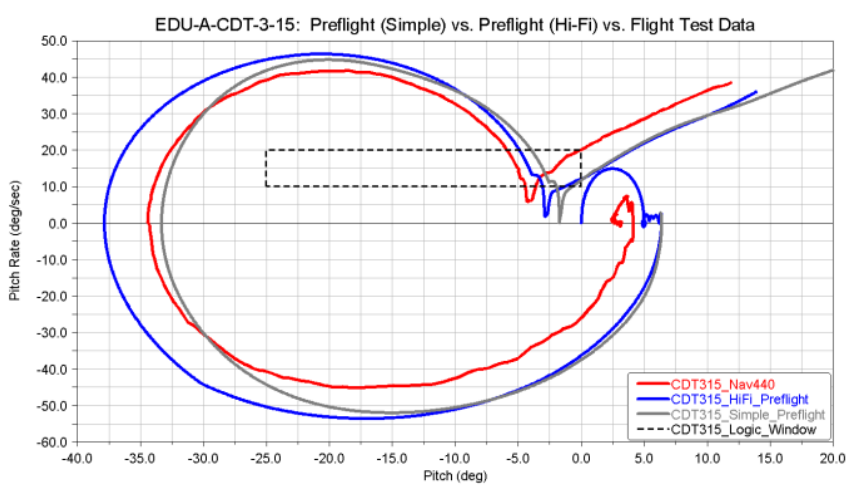

Figure 29: CDT315 Dynamics Summary provide loads data that are to be used to anchor the Hi-Fi model with the objective of using it for predictions to enable extractions from 35,000-ft in subsequent tests. The Hi-Fi delivered exceptional loads predictions as is shown Figure 15.

The gray trace in Figure 29, shows the preflight prediction produced by the primary simulation that was used in the smart separation window definition. The blue trace shows the preflight prediction of the $\mathrm{Hi}-\mathrm{Fi}$ model. The simulations coincided at a pitch rate of $0 \% \mathrm{~s}$ and pitch angle of $5^{\circ}$, then diverged with the simplified model tracking the test data more closely until the positive pitch rate peaked near $40 \mathrm{deg} / \mathrm{sec}$, after which the two models began to converge again prior to entering the design separation window from the top of the right side. The Hi-Fi model entered the window closer to the test point, despite its minimum pitch angle being significantly less than the test value. Both predictions generally bounded the dynamics experienced in flight.

American Institute of Aeronautics and Astronautics 


\section{Challenges with Modeling \& Testing Extraction Techniques}

The Adams extraction-separation model was originally developed to optimize the conditions for a PTV/CPSS separation window and determine the PTV state vector at separation. As the CPAS EDU test campaign progressed, the test technique evolved from a standard load transfer extraction to a backstop reposition extraction. The demand for loads predictions was required to perform safety margin assessments for components in the systems' load path during the backstop-reposition event. Modeling capabilities produced representative static loads when the system reached equilibrium, but was not sophisticated enough to account for transient loads experienced during the highly dynamic event at ramp clear. Since the first backstop reposition test, EDU-A-CDT-3-10, was successful there was no precedence for a high fidelity model. Safety margin in the hardware components were primary positive and used Adams static loads predictions produced with a simplified model to assess failure modes.

\section{A. CPSS Backstop Rigging Configuration}

The CPAS backstop configuration evolved to meet the changing testing objectives. As a result, new components were added and the rigging process was tuned to account for the test-to-test variations. Figure 30 is intended to provide a snapshot of how the rigging of the extraction system evolved from EDU-A-CDT-3-10 to EDUA-CDT-3-15. The MARM was eventually removed from the configuration and 6:2 confluence was introduced as the new standard for executing a backstop reposition technique. Extraction parachutes are now released using single event fitting cutters located at the four corners of the backstop.

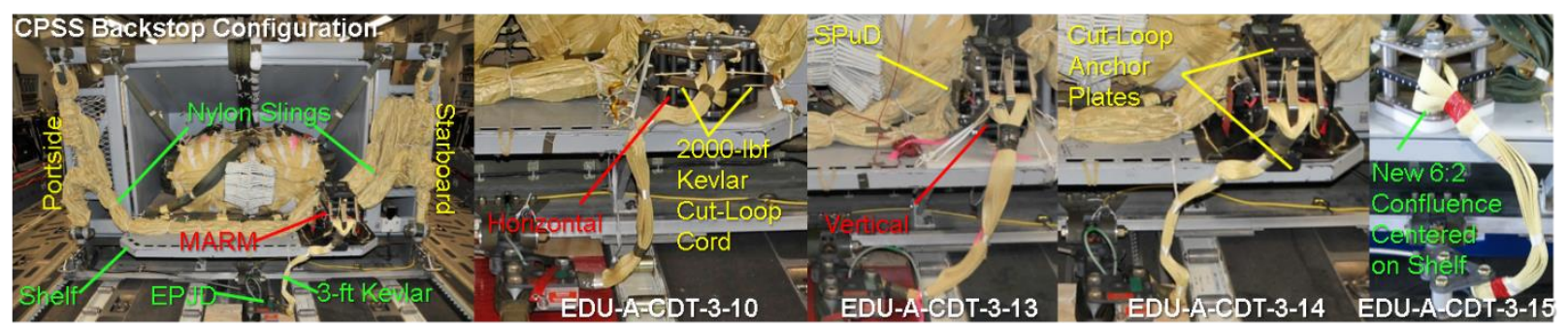

Figure 30: CPSS Backstop Rigging Variation

\section{B. EDU-A-CDT-3-13 (Test Failure Case 1)}

The first backstop reposition malfunction occurred at 13,000-ft. The Mid-Air Release Mechanism (MARM) prematurely released the backstop Nylon slings and static-line deployed the Recovery Mains and Stability parachutes at aircraft extraction. The MARM experienced high accelerations during the high energy event and the 2000-lbf Kevlar cut cord used in the MARM rigging process failed, releasing the cut-loop anchor plates, thus allowing the slings and Extraction parachutes to be released. The smart separation time window was programmed from $1.0 \mathrm{~s}-\mathrm{RC}$ to $4.0 \mathrm{~s}-\mathrm{RC}$ and the separation event occurred at the close of the window. The PTV separated heat shield forward and was able to recover from the malfunction. With the loss of the extraction parachute pull-force the mated vehicle pitch angle reached $20^{\circ}$ at a rate of $12 \%$ as shown in Figure 31. The loss of pull-force is seen in the loads trajectory at $0 \mathrm{~s}-$ RC. At this instant, the Recovery Mains are inflating and required $4.0 \mathrm{~s}$ to reach 35,000-lbf at the close of the window. A summary of the extraction-separation sequence is shown and post-test reconstruction efforts are underway to match the dynamics using the aerodynamic approach (aerodynamics section).

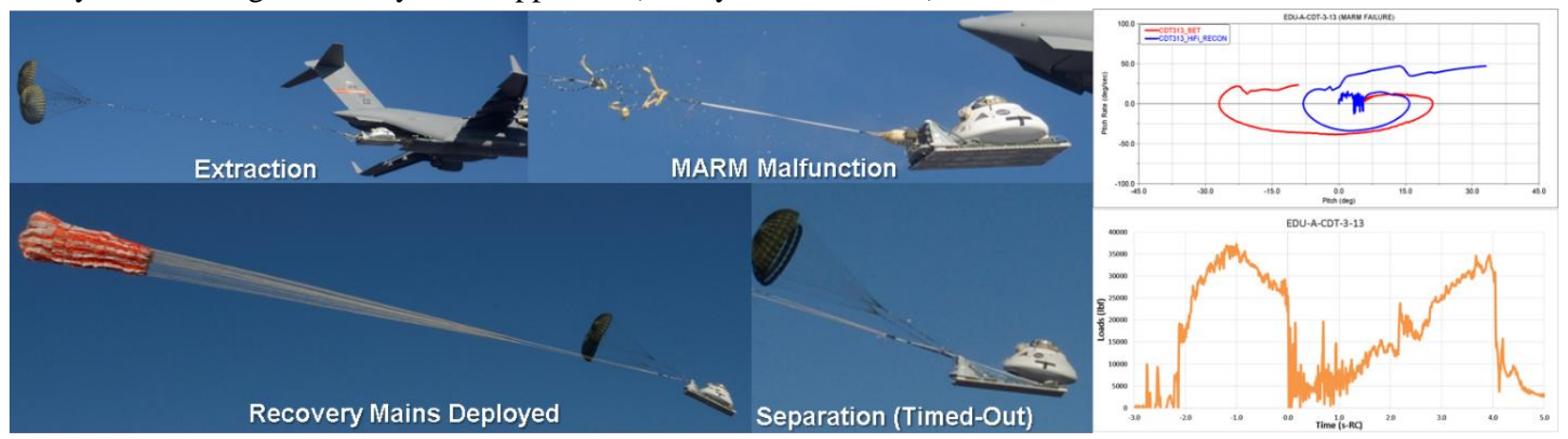

Figure 31: EDU-A-CDT-3-13 Extraction Malfunction at 13,000-ft.

American Institute of Aeronautics and Astronautics 


\section{EDU-A-CDT-3-14 (Test Failure Case 2)}

The second backstop reposition failure occurred at 35,000-ft. Modeling capabilities were not at a state to keep up with the test operations team analysis demands. Any analysis solutions would require extensive model developments that did not align with an aggressive test schedule. Thus, the previous failure was addressed with a hardware change to the MARM that included heavier cut-loop anchor plates and the replacement of mechanical cutters with electronic cutters (SPuD) to allow the CPSS to fall under the Extraction parachutes for a longer duration. Following the second failure, the PTV separated apex forward with a pitch angle of $-25^{\circ}$ and rate of $-11^{\circ} / \mathrm{s}$ at the logic window close time (5.0 s-RC). All GPS antenna located on the parachute compartment tunnel were destroyed by the programmer harness slings sweeping across the tunnel. By programmer inflation, the PTV attitude reached $-40^{\circ}$ and $-50 \%$. Fortunately, the programmer parachutes regained control authority over the unstable PTV and damped out the dynamics prior to the release of the FBC and $10 \mathrm{~s}$ free-fall phase. A summary of the extraction-separation test sequence is shown in Figure 32. A failure investigation team was formed and attributed the prematurely activated electronic cutters and 2000-lbf Kevlar cut-loop cord as probable contributors to the malfunction. An extensive Adams modeling upgrade effort was initiated as a result and a summary of the upgrades are described in the preceding sections.
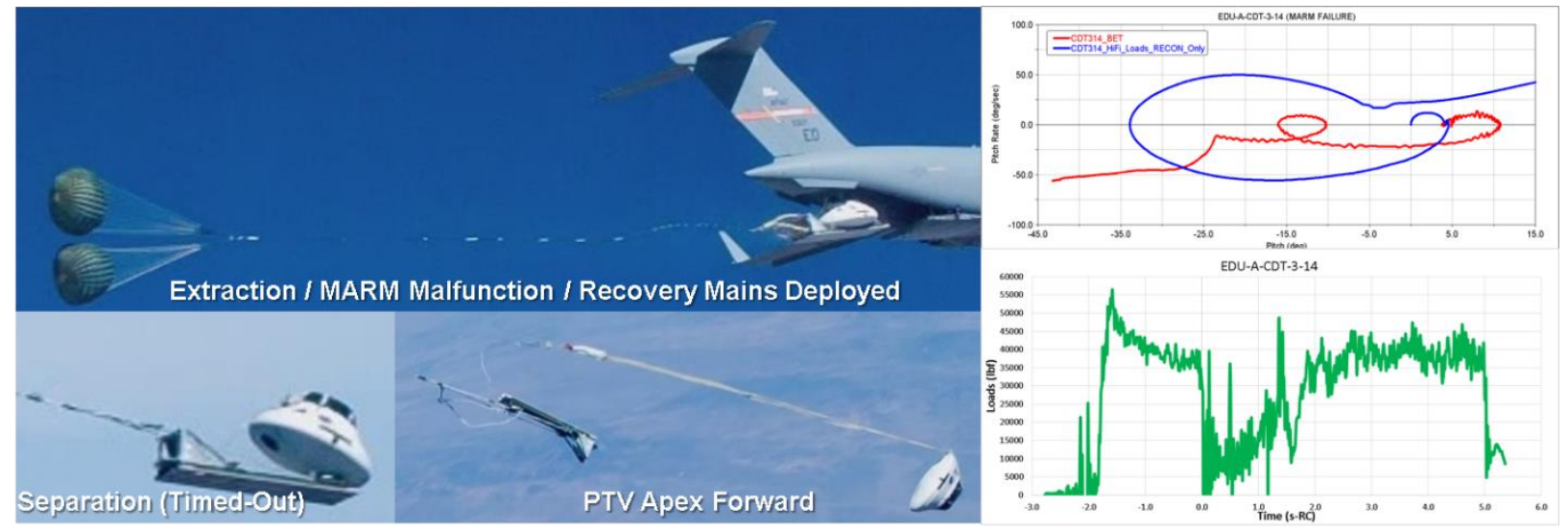

Figure 32: EDU-A-CDT-3-14 Extraction Malfunction at 35,000-ft

\section{Conclusion}

The authors of this paper have relentlessly searched for physics based solutions to anchor the Adams simulation with to produce exceptional loads and dynamics preflight predictions and post-test reconstructions. Numerous upgrades have been undertaken in response to identified modeling deficiencies. Representative hardware components have been introduced into the simulations extraction system and a finite element approach has been integrated to account for the mass of lines. A novel non-linear force function has been introduced to model the performance of specific CPAS lines with varying lengths and material properties. The finite element approach along with the nonlinear force function help produce higher fidelity loads predictions with a focus on transient loads. Prior to this upgrade, only static loads could be produced by the Adams simulation.

An aerodynamic and external force approach has been explored to produce flight correlations. The use of pre and post ramp clear external forces help produce good dynamics correlations but at the cost of lower fidelity loads predictions. Aerodynamic parameters representing vehicle pitch damping derivatives have been tuned with success to match flight dynamics while maintaining exceptional loads predictions of CPAS tests. The true answer for matching flight dynamics lies between aerodynamics and external force influences, but tuning aerodynamic parameters is the current method adopted for post-test reconstructions.

Three types of damping have been introduced into the simulation to remove simulation artifacts, numerical instabilities and unrealistic oscillations in hardware components and lines. Caution must be taken when applying damping terms to ensure the analyst does not over-damp the system and change the dynamics of the simulation and system being analyzed.

Two failure cases have been experienced during the CPAS EDU testing campaign and break-through developments have arisen from the lessons learned in an operations and analysis perspective. The project overcame this challenge with the execution of EDU-A-CDT-3-15, which successfully used the backstop reposition test technique and is planning a 35,000-ft test to meet upcoming objectives required for qualification of the parachute system. 


\section{References}

${ }^{1}$ Ibrahim, S.K. and Engdahl, R.A., "Parachute Dynamics and Stability Analysis," 1 February 1974. Honeywell INC, Minneapolis, Minnesota, 55413, NASA-CR-120326.

${ }^{2}$ Knacke, T.W., "Parachute Recovery Systems Design Manual," U.S. Navy Report NWC TP-6575, Para Publishing Co., Santa Barbara, CA, 1992.

3 Ray, E.S., Varela, J.G., "Inflation of Unreefed and Reefed Extraction Parachutes," $23^{\text {rd }}$ AIAA Aerodynamics Decelerator Systems Technology Conference, Daytona, FL, March 2015 (submitted for publication)

${ }^{4}$ Fraire, U., Anderson, K, and Cuthbert, P.A., "Extraction and Separation Modeling of Orion Test Vehicles with ADAMS Simulation”, 22 $2^{\text {nd }}$ AIAA Aerodynamic Decelerator Systems Technology Conference, Daytona, FL, March 2013, AIAA Paper 2013-1394

${ }^{5}$ Stuart, Philip, and Bourland, Gary, W.,"CPAS Aerodynamic Database Application Programming Interface User's Guide,” Version 3.2.0, CPAS Analysis IPT, NASA-JSC EG Document, December 2013.

${ }^{6}$ Reddy, Satish, "CPAS Nylon Confluence Backstop - Load Analysis -20\% elongation, load shared by 2 straps,” JSC Engineering, Technology \& Science (JETS) - Jacobs Engineering, September 2014.

${ }^{7}$ Macioce, Paul, “Viscoelastic Damping 101,” Roush Industries, Accessed January 2015.

${ }^{8}$ Foss, Gary, C., "Modal Damping Estimates from Static Load-Deflection Curves," Structural Dynamics Laboratory, Boeing Commercial Airplane Group, Accessed January 2015.

${ }^{9}$ Ray, Eric, “EDU-CDT-3-11 Test Report,”. JETS - Jacobs Engineering 